\title{
On the Nonlinear Evolution of a Stationary Cross- Flow Vortex in a Fully Three-Dimensional Boundary Layer Flow
}

\section{J.S.B. Gajjar}

Institute for Computational Mechanics in Propulsion

Cleveland, Ohio

and University of Manchester

Manchester, United Kingdom

October 1995

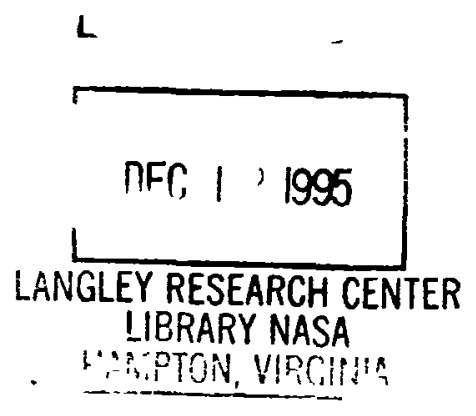

Prepared for

Lewis Research Center

Under Cooperative Agreement NCC3-370

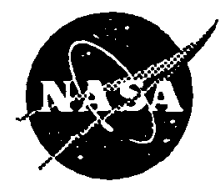

National Aeronautics and Space Admınistration
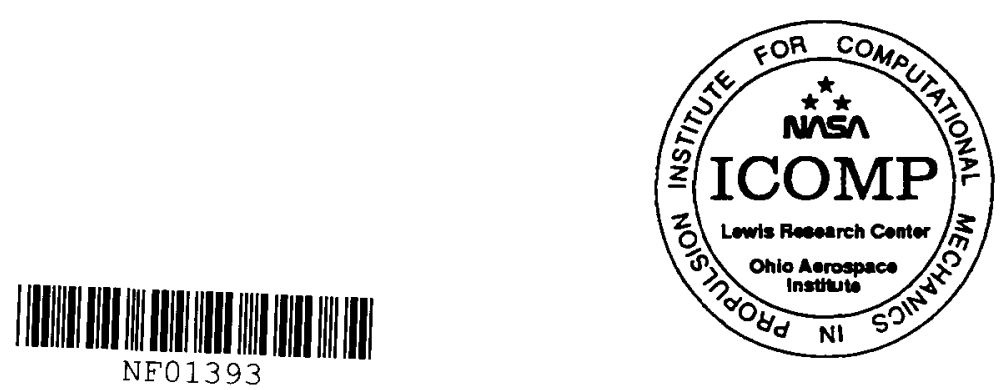


\title{
On the nonlinear evolution of a stationary cross-flow vortex in a fully three-dimensional boundary layer flow.
}

\author{
J.S.B. Gajjar \\ Institute for Computational Mechanics in Propulsion \\ Cleveland, Ohio 44135 \\ and University of Manchester \\ Department of Mathematics \\ Manchester M13 9PL, United Kingdom
}

\begin{abstract}
We consider the nonlinear stability of a fully three-dimensional boundary layer flow in an incompressible fluid and derive an equation governing the nonlinear development of a stationary cross-flow vortex The amplitude equation is a novel integro-differential equation which has spatial derivatives of the amplitude occurring in the kernal function. It is shown that the evolution of the cross-flow vortex is strongly coupled to the properties of an unsteady wall layer which is in fact driven by an unknown slp velocity, proportional to the amplitude of the cross-flow vortex. The work is extended to obtain the corresponding equation for rotating disk flow. A number of special cases are examined and the numerical solution for one of cases, and further analysis, demonstrates the exustence of finite-distance as well as focussing type singularities. The numerical solutions also indicate the presence of a new type of nonlinear wave solution for a certain set of parameter values.
\end{abstract}




\section{Introduction}

One of the earliest experimental and theoretical investigations of the stability of thieedimensional (3D) boundary layers was conducted by Giegory. Stuart \& Walker (195j). (hereafter referred to as GSW). The boundary layer flows studied were the flow over a rotating disk, and the flow over swept wings. Using a china-clay visualisation technque they were able to demonstrate the presence of a highly iegular, stationary, pattern of vortices spaced equally around the disk, or along the surface of the wing In addition. with the and of a microphone probe, they were able to detect travelling waves close to the surface of the disk. Stuart in GSW suggested that the instabilities could be explained in terms of the inflexional character of the effective mean velocity profile in certain directions, ( the term effective mean velocity profile refers to a certain linear combination of the streamwise and spanwise velocity components) According to his suggestion the stationary pattern was that associated with the inviscid instability of the velocity profile which had a zero at a point of inflexion. The non-stationary, or travelling wave pattern, could also be explained in terms of the inviscıd instabilty of the mean flow. These instabilities are now commonly referred to as cross-flow instabilities and their importance stems from the fact that they arise naturally in many fully three-dimensional boundary layer flows of practical importance, such as in the flow past swept arrcraft wings, rotating flows, and so on.

The experimental observations of GSW have been confirmed in many subsequent experıments on rotating disk flow, see for instance Malık et al. (1981), Kohama (1984). Kobayashi et al. (1980), Kohama et al. (1991), as well in experiments on swept wings and cylinders, Poll (1985), Michel et al. (1985), Mulleı \& Bippes (1988), Saric el. (1989). An extensive review of many aspects of the instability of 3D boundary layers including cross-flow instability, and covering many of the early investigations, may be found in Reed \& Saric (1989), see also Arnal (1986).

The more recent experiments on cross-flow instabllity hare considered nonlinear effects and in Kohama (1984), Kohama et al (1991) an explosive secondary instability, which takes the form of ring-like vortices wrapped around the primary cross-flow vortex, has been observed. In the experiments of Muller \& Bippes (1988) it has been found that the stationary as well as the non-stationary vortices attained a nonlinear saturation amplitude. A similar phenomenon has been observed in the direct numerical simulations of Meyer \& Kleiser (1988), and also Malik (1986), Malık \& Li (1992), Malık et. al. (1994). using more approximate methods based on the parabolised stability equations approach

There are very few self-consistent theoretical investigations of the stability of crossflow vortices. Hall (1986) extended Stuart's analysis to compute correction terms for the number of vortices as well as the inclination of the stationary vortices in the limit of large Reynolds numbers. He was able to explain the existence of another branch of the neutral curve, calculated previously by Malık (1986). as being associated with a mode with zero shear stress. Mackerrel (1987) extended Hall's work to derive a weakly nonlinear amplitude equation for the zero shear stress mode. Bassom \& Gajjar (1989) studied the linear and nonlinear neutral stability properties of long wavelength nonstationary cross-flow vortices using scalings appropriate to the upper-branch stability of a two-dimensional boundary layer. Recently Gajjai (1994). (1995), has extended the Bassom \& Gajjar (1989) work to compressible flows and looked at non-neutral modes

The main objective of this paper is to present a self-consistent theoretical description 
of the nonlineal evolution of a stationary cross-flow voltex. In companion papers Gajuar \& Arebi (1995) this wolk is extended to the non-stationaiy case and in Gajjar \& Sibanda (1995) to compressible flows We study a general 3D boundary layer flow and consider a flow direction in which the effective relocity profile has a zero at a point of inflexion. giving rise to the stationary closs-flow vol tex. and include modulation in time and space. The stability analysis leads to a novel integro-differential equation for the amplitude of the cross-flow vortex with spatial derıvatives of the amplitude occurning in the kernal function.

The starting point for the analysis is to consider a stationary cross-flow vortex in a neutral or near neutral state Thus with small growth rates, and since the basic instabilty mechanısm is Rayleigh instability, GSW (1955), we may appeal to the ideas of unsteady nonlinear critical layer theory used successfully in many studies of the instabilities of planar shear and boundary layer flow's, see for example Hickernell (1984), Goldstein \& Leib (1989), Leib(1991), Goldstein \& Choi (1989), Wu(1992), Wu (1994). The application of unsteady nonlinear critical layer theory to shear flow and boundary layer instabilities, and a discussion of the properties of some of the integro-differential amplitude equations which arise in this type of work, may be found in the excellent reviews by Cowley \& Wu (1994) and Goldstein (1994)

Our analysis is similar to that of Wu (1994) who investigated the stability of a twodimensional Stokes-Layer to 3D disturbances and studied the effect of slow temporal and spanwise modulation The major difference in the present work and that of Wu (1994) arises from the three-dimensionality of the basic flow used here This leads to a different amplitude equation with single spatial derivatives of the amplitude in the integro-differential operator and the kernal function, as opposed to the double derivatives in Wu's work. Another important aspect of the current work is the coupling of the evolution of the disturbance amplitude with the properties of an unsteady wall layer. This extra new feature arises primarily because of the scalings associated with a stationary cross-flow vortex.

The basic scaling used in the analysis below may be derived using an argument similar to that first used by Hickernell (1984), see also Wu (1994). We present some of the detalls of this argument as there are a number of important differences from those given in Wu (1994). Consider a cross-flow vortex of amplitude $\delta$. We need to determine the size of $\delta$ such that critical layer nonlinearity affects the amplitude of the cross-flow vortex. We allow for slow temporal and spatial modulation with respective relative scales $\Delta_{T}, \Delta_{Z}$. The suffix $Z$ here denotes variations in the direction normal to the Squire direction Since the wavenumbers are of magnitude $O\left(R^{\frac{1}{2}}\right), R$ being the Reynolds number, a balance of the inertial and viscous terms shows that the thickness of the cuitical layer at $Y_{c}$ is given by $Y-Y_{c}=O(\epsilon)$ where $Y$ denotes the $O(1)$ boundary layer coordinate, and $\epsilon=R^{-\frac{1}{6}}$. Thus the spanwise component of the disturbance velocity, $w$, has a pole singularity of size

$$
w=O\left(\frac{\delta}{\left(Y-Y_{c}\right)}\right) .
$$

In $\mathrm{Wu}(1994)$ the equivalent $w$ is much smaller since

$$
w=O\left(\frac{\frac{\partial p}{\partial Z}}{\left(Y^{*}-Y_{c}\right)}\right)=O\left(\frac{\delta \unlhd_{z}}{\left(I^{*}-Y_{c}\right)}\right)
$$

there. The relation ( $\left.\begin{array}{ll}1 & 1\end{array}\right)$ stems from a different balance Fiom the continuity equation 
the Squire component of velocity $u$ is then of size

$$
u=O\left(u_{z}\right)=O\left(\frac{\delta \Delta_{z}}{\left(I^{\circ}-I_{c}^{*}\right)}\right)
$$

Again an important difference between the analy sis here and in Wu (1994) is noted, since the equivalent expression there is

$$
u=O\left(\frac{\frac{\partial^{2} p}{\partial Z^{2}}}{\left(Y-Y_{c}\right)}\right)=O\left(\frac{\delta \Delta_{Z}^{2}}{\left(Y-I_{c}\right)}\right)
$$

This is the reason why the final amplitude equation in Wu's work involves double derivatives in $Z$ whereas here there are single derivatives in $Z$

The remainder of the argument follows closely that given in Hickernell (1984) and Wu (1994), and shows that

$$
\Delta_{T}=\Delta_{Z}=O\left(Y-Y_{c}\right)=O(\epsilon),
$$

for unsteadiness and spanwise modulation to produce a non-equilibrium effect in the critical layer. Interactions inside the critical layer give rise to a Squire component of velocity of order

$$
O\left(\frac{\delta^{3} \Delta_{Z}}{\Delta_{T}^{2}\left(Y-Y_{c}\right)^{3}}\right)
$$

and this affects the outer flow provided

$$
O\left(\frac{\delta^{3} \Delta_{Z}}{\Delta_{T}^{2}\left(Y-Y_{c}\right)^{3}}\right)=O\left(\delta \Delta_{T}\right)
$$

Thus using (1.2), (1.3) we find that $\delta=O\left(\epsilon^{\frac{5}{2}}\right)$.

In section 2 below the problem is formulated The detarls of the outer inviscid flow are considered in section 3 where a solvability condition, which leads to the amplitude equation, is derived. The solvability condition shows that the amplitude of the cross-flow vortex depends on the displacement induced by the wall lajer as well as the jump across the critical layer In sections 4 and 5 the solutions inside the critical layer are obtained and these are then used to determine the nonlinear jump conditions which appear in the solvability condition. The amplitude equation is obtained in section 5 . In section 6 the properties of this amplitude equation are discussed and some results are presented Finally we conclude with additional comments in section 7 . In Appendix A the analysis is extended to the obtain the corresponding amplitude equation for a stationary cross-flow vortex in the flow over a rotating disk

Throughout this work the fluid is taken to be incompressible and the Reynolds number to be large. 


\section{Problem formulation}

Consıder cartesian coordinates $(\bar{x}, y, \bar{z})$ non-dimensionalised with respect to a lengthscale $L$ and where $x$ is in the streamwise duection, $\bar{z}$ is in the spanwise direction and $y$ is normal to the body The corresponding non-dimensional velocity components are $(\bar{u}, v, \bar{w})$. It is convenient to work in terms of Squire coordinates defined by

$$
x=\alpha \bar{x}+\beta \bar{z}, \quad z=-\beta \bar{x}+\alpha \bar{z}
$$

with corresponding velocities

$$
u=\alpha \bar{u}+\beta \bar{w}, \quad w=-\beta \bar{u}+\alpha \bar{w}
$$

where $\alpha=\cos \theta, \beta=\sin \theta$ and the angle $\theta$ will be fixed subsequently.

In terms of $x, z$ the Navier-Stokes equations are

$$
\begin{gathered}
\operatorname{div} \mathbf{u}=0, \\
\mathbf{u}_{t}+\mathbf{u} \quad \nabla \mathbf{u}=-\nabla p+\frac{1}{R} \nabla^{2} \mathbf{u}
\end{gathered}
$$

Here $\mathbf{u}=(u, v, w), t$ is the non-dimensional time, $p$ is the non-dimensional pressure, $R=U_{C} L / \nu$ is the Reynolds number, $U_{C}$ is a characteristic velocity scale, and $\nu$ is the kinematic viscosity The Reynolds number $R$ is assumed to be large, and we set $\varepsilon=R^{-1 / 6}$.

\subsection{Basic Flow}

The basic flow is taken to be a fully three-dimensional boundary lay'er flow given by

$$
\begin{aligned}
(u, v, w)= & \left(U_{B}, \varepsilon^{3} V_{B}, W_{B},\right)\left(x, Y^{\prime}, z\right)+\ldots \\
& p=p_{B}(x, z)+\ldots,
\end{aligned}
$$

where $Y=\varepsilon^{-3} y$ is the boundary layer coordinate. The basic flow satisfies the boundary layer equations

$$
\begin{gathered}
U_{B x}+V_{B Y}+W_{B z}=0 \\
U_{B} U_{B x}+V_{B} U_{B Y}+W_{B} U_{B z}=-p_{B x}+U_{B Y Y} \\
U_{B} W_{B x}+V_{B} W_{B Y}+W_{B} W_{B z}=-p_{B z}+W_{B Y Y}
\end{gathered}
$$

with the boundary conditions

$$
U_{B}=V_{B}=W_{B}=0 \text { on } \quad \mathrm{I}=0 .
$$

and

$$
U_{B} \rightarrow U_{B \infty}, \quad W_{B} \rightarrow W_{B \infty} \text { as } I^{-} \rightarrow \infty
$$

Following the discussion in the Introduction, the basic flow $U_{B}$ is such that it has a zero at a point of inflexion Thus near $Y=Y_{c}$ where $U_{B}\left(x, Y_{c}, z\right)=0$ we can expand the basic flow quantities as

$$
\begin{aligned}
U_{B} & =\varepsilon \lambda_{1} \eta+\varepsilon^{3} \lambda_{3} \eta^{3}+ \\
V_{B} & =V_{B 0}^{(0)}+\varepsilon V_{B 0}^{(1)} \eta+\ldots \\
W_{B} & =\mu_{0}+\varepsilon \mu_{1} \eta+\varepsilon^{2} \mu_{2} \eta^{2}+
\end{aligned}
$$


where $I^{\cdot}=Y_{c}^{-}+\varepsilon \eta$ and $\lambda_{1}, \lambda_{3} . I_{B O}^{r(0)}, I_{B O}^{-(1)}, \mu_{0}, \mu_{1} . \mu_{2}$, and all functions of $x$ and $z$.

After substituting (25) into the basic flow equations and equating powers of $z$ the following relations are easily derived.

$$
\begin{gathered}
V_{B 0}^{(0)} \lambda_{1}-\mu_{0} Y_{C z} \lambda_{1}=-p_{B x} \\
V_{B 0}^{(1)} \lambda_{1}+\mu_{0} \lambda_{1 z}-\lambda_{1}^{2} Y_{C x}-\mu_{1} \lambda_{1} Y_{C=}=6 \lambda_{3}, \\
V_{B 0}^{(1)}+\mu_{0=}-Y_{C x} \lambda_{1}-Y_{C z} \mu_{1}=0, \\
V_{B 0}^{(0)} \mu_{1}+\mu_{0} \mu_{0 z}-\mu_{0} Y_{C z} \mu_{1}=-p_{B z}+2 \mu_{2}
\end{gathered}
$$

These relations are used later in section 4. The properties of the basic flow near the wall are also needed later and for $Y$ small we have

$$
\begin{aligned}
U_{B} & =\bar{\lambda}_{1} Y+\ldots \\
W_{B} & =\bar{\mu}_{1} Y+\ldots
\end{aligned}
$$

Since the velocity profile $U_{B}$ is zero at the wall and has a zero somewhere in the flow, it is clear that $\lambda_{1}$ and $\bar{\lambda}_{1}$ have opposite signs.

Next we consider a stationary cross-flow vortex with a wavenumber $\gamma$ (scaled with respect to boundary-layer thickness), in the $x$ direction. and introduce additional coordinates $X, Z, T$ to allow for modulation such that,

$$
\begin{gathered}
\frac{\partial}{\partial x} \rightarrow \varepsilon^{-3}\left[\frac{\partial}{\partial \xi}+\varepsilon \frac{\partial}{\partial X}\right]+\frac{\partial}{\partial x} \\
\frac{\partial}{\partial z} \rightarrow \varepsilon^{-2} \frac{\partial}{\partial Z}+\frac{\partial}{\partial z}, \quad \frac{\partial}{\partial t} \rightarrow \varepsilon^{-2} \frac{\partial}{\partial T}
\end{gathered}
$$

The $\varepsilon^{-3}$ factors above account for the scaling with respect to boundary layer thickness and the $\frac{\partial}{\partial x}, \frac{\partial}{\partial z}$ variations are needed to account for the local variations of the mean flow in the $x$ and $z$ coordinate directions.

\section{Solution outside the critical layers}

\subsection{Main part of the boundary layer}

We first consider the solution in the main part of the boundary layer where $Y=\varepsilon^{-3} y$ is $O(1)$ In this region the expansions for the flow quantities are

$$
\begin{aligned}
& u=U_{B}+\delta\left(\bar{u}_{0}+\varepsilon \bar{u}_{1}+\right) \\
& v=\quad \delta\left(\bar{v}_{0}+\varepsilon \bar{v}_{1}+\right) \\
& w=W_{B}+\delta\left(\bar{w}_{0}+\varepsilon \bar{w}_{1}+.\right) \\
& p=p_{B}+\delta\left(\bar{p}_{0}+\varepsilon \bar{p}_{1}+\right)
\end{aligned}
$$

where the disturbance size $\delta$ will be taken to be $O\left(\varepsilon^{5 / 2}\right)$ subsequently, but since the properties outsıde the critical layers are largely dictated by lineai dynamics, it is convenient to work with $\delta$ 
After substituting (3 1 ) into the Navier-Stokes equations (2 3) and using (2.8), the leading ordeı disturbance equations are found to be

$$
\begin{gathered}
\bar{u}_{0 \xi}+\bar{v}_{0 Y}=0, \\
\tau_{B} \bar{u}_{0 \xi}+\bar{v}_{0} U_{B I}=-\bar{p}_{0 \xi}, \\
U_{B} \bar{w}_{O \xi}+\bar{v}_{0} W_{B \perp}=0
\end{gathered}
$$

The problem (3.2) is just that for the stationary cross-flow vortex, Gregory, Stuart \& Walker (1955), and if we set

$$
\begin{aligned}
& \overline{u_{0}}=A \hat{U}_{0}(Y, x, z) e^{\imath \gamma \xi}+\mathrm{c} c . ., \\
& \overline{v_{0}}=-\imath A \tilde{V}_{0}(Y, x, z) e^{\imath \gamma \xi}+\mathrm{c} . . . \\
& \overline{w_{0}}=A \tilde{W}_{0}(Y, x, z) e^{\imath \gamma \xi}+\text { c.c.. } \\
& \overline{p_{0}}=A \tilde{P}_{0}(Y, x, z) e^{\imath \gamma \xi}+\text { c.c. }
\end{aligned}
$$

where c.c.. denotes the complex conjugate, then $\tilde{V}_{0}$ satısfies Rayleigh's equation

$$
\tilde{V}_{O Y Y^{\prime}}-\left(\gamma^{2}+\frac{U_{B Y Y}}{U_{B}}\right) \tilde{V}_{0}=0
$$

with the boundary conditions

$$
\begin{gathered}
\tilde{V}_{0}=0 \quad \text { on } \quad Y=0, \\
\left|\tilde{V}_{0}\right|<\infty \quad \text { as } \quad Y \rightarrow \infty
\end{gathered}
$$

In (3.3) $A(X, T, Z)$ is the normalised amplitude of the cross-flow rortex and dependent on the slow scales $X, T$ and $Z$. The eigenfunctions ${ }^{1}$ in (3 3) depend on $x$ and $z$ because of the basic flow $U_{B}$ The problem (3.4) has to solved with the additional constraints

$$
U_{B}=0=U_{B Y^{\prime} Y} \quad \text { at } Y=Y_{c}^{\circ} .
$$

so that the point $Y=Y_{c}$ is a regular point of $(34 \mathrm{a})$. The conditions (3.4d) fix the angle $\theta$ and $Y_{c}$ since

$$
U_{B}=\cos \theta \bar{U}_{B}+\sin \theta \bar{W}_{B} .
$$

Given $\theta$ and $Y_{c}(34 \mathrm{a}, \mathrm{b}, \mathrm{c})$ then determines the wavenumber $\gamma$ of the stationary cross-flow vortex and the eigenfunction $\tilde{V}_{0}$. and $\tilde{U}_{0}, \tilde{W}_{0}$ are obtained fiom

$$
\begin{gathered}
\tilde{U}_{0}=\frac{1}{\gamma} \tilde{V}_{0 Y^{\prime}}, \\
\tilde{V}_{0}=\frac{W_{B Y} \cdot \hat{V}_{0}}{\gamma U_{B}}
\end{gathered}
$$

In general the problem for $\tilde{V}_{0}$ has to be solved numerically but the solution properties near $Y=Y_{c}$ can be obtained using a Frobenius expansion. Thus near $Y=Y_{c}$ the solution for $\tilde{V}_{0}$ can be expressed as

$$
\hat{V}_{0}=C^{ \pm} \phi_{a}+B^{ \pm}\left[\phi_{b}+p_{j} \phi_{a} \ln |\bar{\eta}|\right]
$$

\footnotetext{
${ }^{1}$ The amplitude $\mathrm{A}$ also depends on $x$ and $z$ as can be seen from the amplitude equation in section 5 below, but since the dependence is only parametric it is not shown explicitly for the sake of clarity
} 
where

$$
\begin{gathered}
\phi_{a}=\bar{\eta}+\frac{1}{2} p_{j} \bar{\eta}^{2}+. . \\
\phi_{b}=1+q, \bar{\eta}^{2}+.
\end{gathered}
$$

are two linearly independent solutions of Rayleıgh's equatıon and $\bar{\eta}=Y-Y_{c}$

Note that

$$
p_{\jmath}=\frac{2 \lambda_{2}}{\lambda_{1}}=0, \quad q_{3}=\frac{1}{2} \gamma^{2}+\frac{3 \lambda_{3}}{\lambda_{1}} .
$$

The constants ${ }^{2} C^{+}-C^{-}, B^{+}-B^{-}$will later be shown to be equal to zero

From (3.5b) it follows that $\tilde{W}_{0}$ has a pole singulanty near $I_{c}^{-}$if $\mu_{1} \neq 0$.

The second order problem for the flow quantities leads to the equations

$$
\begin{gathered}
\bar{u}_{1 \xi}+\bar{u}_{0 X}+\bar{v}_{1 Y}+\bar{w}_{0 Z}=0 \\
\bar{u}_{0 T}+U_{B}\left(\bar{u}_{1 \xi}+\bar{u}_{0 X}\right)+\bar{v}_{1} U_{B Y}+U_{B} \bar{u}_{0 Z}=-\bar{p}_{1 \xi}-\bar{p}_{0 X} \\
\bar{v}_{0 T}+U_{B}\left(\bar{v}_{1 \xi}+\bar{v}_{0 X}\right)+W_{B} \bar{v}_{0 Z}=-\bar{p}_{1 \zeta} \cdot \\
\bar{w}_{0 T}+U_{B}\left(\bar{w}_{1 \xi}+\bar{w}_{0 X}\right)+\bar{v}_{1} W_{B Y}+W_{B} \bar{w}_{0 Z}=-\bar{p}_{0 Z} .
\end{gathered}
$$

Thus using (3.7) it is found that the $e^{\imath \gamma \xi}$ component of ${\overline{v_{1}}}_{1}{\hat{V_{1}}}_{1}$ satisfies the equation

$$
\begin{gathered}
U_{B}\left(\tilde{V}_{1 Y Y}-\gamma^{2} \tilde{V}_{1}\right)-\tilde{V}_{1} U_{B Y Y}=\frac{1}{\gamma} \frac{U_{B Y Y}}{U_{B}^{\top}} \tilde{V}_{0} \frac{\partial A}{\partial T} \\
-\frac{1}{\gamma} \frac{\partial A}{\partial Z}\left(\frac{W_{B Y \zeta}}{U_{B}}-\frac{W_{B} U_{B I Y}}{U_{B}^{2}}\right) U_{B} \tilde{V}_{0}-2 \gamma \frac{\partial A}{\partial{ }_{1}} \tilde{V}_{0} U_{B} .
\end{gathered}
$$

The solution for $\tilde{V}_{1}$ near $Y=Y_{c}^{*}$ is easily obtained and takes the form

$$
\tilde{V}_{1} \sim g_{ \pm} \phi_{a}+f_{ \pm} \phi_{b}+\left[\frac{B^{ \pm}}{\gamma}\left(\frac{6 \mu_{0} \lambda_{3}}{\lambda_{1}^{2}}-\frac{2 \mu_{2}}{\lambda_{1}}\right) \frac{\partial A}{\partial Z}+\frac{6 \lambda_{3} B^{ \pm}}{\gamma \lambda_{1}^{2}} \frac{\partial A}{\partial T}\right](\eta(\ln |\eta|-1)+\ldots
$$

Note that since $\tilde{V}_{1}$ satisfies the same equation as $\tilde{V}_{0}$ but with a different right hand side, a solution exists only provided a certain solvability condition is satısfied The solvability condition is obtained by multiplying (3.8) by $\tilde{V}_{0}$ and integrating from $Y=0$ to $Y=\infty$ and using (34) This gives

$$
\left[\tilde{V}_{0 Y} \cdot \tilde{V}_{1}-\tilde{V}_{0} \tilde{V}_{1 Y}\right]_{Y_{c}+}^{Y_{c+}+}+\left[\tilde{V}_{0 Y} \tilde{V}_{1}\right]_{Y=0}=\frac{I_{1}}{\gamma} \frac{\partial A}{\partial T}+\frac{I_{2}}{\gamma} \frac{\partial A}{\partial Z}+\gamma I_{3} \frac{\partial A}{\partial X}
$$

where

$$
\begin{gathered}
I_{1}=f_{0}^{\infty} \frac{U_{B Y Y}}{U_{B}^{2}} \tilde{V}_{0}^{2} d Y, \\
I_{2}=-f_{0}^{\infty}\left(\frac{W_{B Y Y}}{U_{B}}-\frac{W_{B} U_{B Y I}}{U_{B}^{2}}\right) \tilde{V}_{0}^{\prime 2} d I \\
I_{3}=-2 \int_{0}^{\infty} \tilde{V}_{0}^{2} d Y
\end{gathered}
$$

The equation (310) is the amplitude equation for the cross-flow vortex and the first term involving the jumps is obtained from an analysis of the critical layer The $\left.\tilde{V}_{1}\right|_{Y=0}$ contribution to the amplitude equation is determined from an analysis of the wall layer, and this is considered next

\footnotetext{
${ }^{2}$ These are again functions of $x$ and $z$ but since the dependence on $x=1$ s parametric we will use the term constants
} 


\subsection{Wall layer analysis}

From $(2.7),(3.1),(32)$, it follows that in the wall layer where $y=\varepsilon^{4} \bar{Y}$ the expansions are

$$
\begin{aligned}
u & =\varepsilon \bar{\lambda}_{1} \bar{\Gamma}+.+\delta\left(\bar{u}_{0}+.\right) \\
u & =\delta\left(\varepsilon \bar{\tau}_{0}+.\right) \\
p & =p_{B}+\delta\left(\varepsilon \bar{p}_{0}+. .\right) \\
w & =\varepsilon \bar{\mu}_{1} \bar{Y}+. .+\delta\left(\bar{w}_{0}+.\right)
\end{aligned}
$$

Substitution into the Navier-Stokes equations then leads to

$$
\begin{aligned}
& \bar{u}_{0 \xi}+\bar{v}_{0 \bar{Y}}=0 \text {, } \\
& \bar{u}_{0 T}+\bar{\lambda}_{1} \bar{Y}^{\bar{u}} \bar{u}_{0 \xi}+\bar{v}_{0} \bar{\lambda}_{1}=-\bar{p}_{0 \xi}+\bar{u}_{1 Y Y}, \quad 0=-\bar{p}_{0 \bar{Y}}, \\
& \bar{w}_{0 T}+\lambda_{1} \bar{\digamma} \bar{w}_{0 \xi}+\bar{v}_{0} \bar{\mu}_{1}=\bar{w}_{01}{ }^{\prime} \text {. }
\end{aligned}
$$

These equations have to be solved subject to the no slip conditions

$$
\bar{u}=\bar{v}=\bar{w}=0 \text { on } \bar{Y}=0
$$

and

$$
\begin{gathered}
\bar{u}_{0} \rightarrow \frac{A(X, Z, T)}{\gamma} \tilde{V}_{0 Y}(Y=0) e^{\imath \gamma \xi}+\text { c c. as } \bar{\Gamma} \rightarrow \infty, \\
\bar{w}_{0} \rightarrow \frac{A(X, Z, T)}{\gamma \bar{\lambda}_{1}} \bar{\mu}_{1} \tilde{V}_{01} \cdot(Y=0) e^{\imath \gamma \xi}+\text { c c. as } \bar{\Gamma} \rightarrow \infty .
\end{gathered}
$$

The displacement from the wall layer provides a contıbution to the amplitude equation and this is given by the finite part of $\bar{v}_{0}$ as $\bar{Y} \rightarrow \infty$. Hence the required matching condition is

$$
\tilde{V}_{1}(Y=0)=\lim _{Y \rightarrow \infty}\left[\bar{v}_{0}+\bar{Y}\left(\imath A(X, Z, T) \tilde{V}_{0 Y}\left(Y^{-}=0\right) \epsilon^{\imath\urcorner \xi}+\text { c.c. }\right)\right] .
$$

The solution of the unsteady wall layer equations (3.13) togethei with the non-slip boundary conditions (3.14a) and slip velocity $(3.14 \mathrm{~b})$ determıne (3.14d). However these equations cannot be solved in isolation because of the unknown slip velocity (3.14b) and in fact the wall layer equations are directly coupled to the nonlinear evolution of the cross-flow vortex amplitude via (3.10).

We consider next the detals of the critıcal layer which determine the unknown jumps in $(3.10)$

\section{Solutions inside the critical layers}

In the critical layer we set $y=\varepsilon^{3} I_{c}+\varepsilon^{4} \eta$ and the outer solutions. with $\delta=\varepsilon^{5 / 2}$, imply the expansions

$$
\begin{array}{r}
u=\varepsilon \lambda_{1} \eta+\varepsilon^{5 / 2} u_{0}+\varepsilon^{3}\left(\lambda_{3} \eta^{3}+u_{1}\right)+\varepsilon^{7 / 2} u_{2}+\ldots \\
v=\varepsilon^{5 / 2} v_{-1}+\varepsilon^{3} V_{B 0}^{(0)}+\varepsilon^{7 / 2} v_{0}+\varepsilon^{8 / 2}\left(v_{1}+I_{B 0}^{r(1)} \eta\right)+\varepsilon^{9 / 2} v_{2}+. \\
w=\mu_{0}+\varepsilon \mu_{1} \eta+\varepsilon^{3 / 2} w_{0}+\varepsilon^{2}\left(\mu_{2} \eta^{2}+w_{1}\right)+\varepsilon^{5 / 2} w_{2}+. \\
p=p_{B}+\varepsilon^{5 / 2} p_{0}+\varepsilon^{7 / 2} p_{1}+\varepsilon^{8 / 2} p_{2}+\varepsilon^{9 / 2} p_{3}+\ldots
\end{array}
$$


After substituting (4 1) into the Nariei-Stokes equations and using the relations (2.6) we obtain the following sequence of equations:

$$
\begin{gathered}
\lambda_{1} v_{-1}=-p_{0 \xi}, \\
0=p_{0 \eta}, \\
u_{0 \xi}+v_{0 \eta}+w_{0 Z}=0, \\
\mathcal{K} u_{0}+v_{0} \lambda_{1}=-\left(p_{1 \xi}+p_{0 x}\right), \\
\mathcal{K} w_{0}+v_{-1} \mu_{1}=0, \\
0=p_{1 \eta},
\end{gathered}
$$

where the operator $\mathcal{K}$ is defined by

$$
\mathcal{K} \equiv \frac{\partial}{\partial T}+\lambda_{1} \eta \frac{\partial}{\partial \xi}+\mu_{0} \frac{\partial}{\partial Z}-\frac{\partial^{2}}{\partial \eta^{2}}
$$

The equations for the second and third order disturbance quantities are

$$
\begin{gathered}
u_{1 \xi}+v_{1 \eta}+w_{1 Z}=0, \\
\mathcal{K} u_{1}+v_{1} \lambda_{1}+v_{-1} u_{0 \eta}=-p_{2 \xi} \\
\mathcal{K} w_{1}+v_{-1} w_{0 \eta}=0, \\
0=-p_{2 \eta},
\end{gathered}
$$

and

$$
\begin{gathered}
u_{2 \xi}+u_{0 X}+v_{2 \eta}+w_{2 Z}-I_{C z} u_{0 \eta}=0, \\
\mathcal{K} u_{2}+\lambda_{1} \eta u_{0 X}+v_{2} \lambda_{1}+v_{-1} u_{1 \eta}+\mu_{1} \eta u_{0 Z} \\
+V_{B 0}^{(0)} u_{0 \eta}+V_{-1} 3 \lambda_{3} \eta^{2}=-\left(p_{3 \xi}+p_{1 X}\right) . \\
\mathcal{K} w_{2}+\lambda_{1} \eta w_{0 X}+v_{-1} w_{1 \eta}+v_{0} \mu_{1}+\mu_{1} \eta w_{0 Z}, \\
+v_{-1} 2 \mu_{2} \eta+V_{B 0}^{(0)} w_{0 \eta}-\mu_{0} \xi_{C=} w_{0 \eta}=-p_{0 Z}, \\
\mathcal{K} v_{-1}=-p_{3 \eta} .
\end{gathered}
$$

The solutions to (4 2)-(4.6) together with matching with the outer flow determine the jumps required for the amplitude equation. These equations are solved $i n$ serzatum using the well established Fourier-Transform technique of Hickernell (1984) It is convenient to introduce a change of variables with

$$
\text { - } \tilde{Z}=\mu_{0}^{-1} Z \quad, \quad \tilde{T}=\mu_{0} T-Z
$$

so that the operator $\mathcal{K}$ becomes

$$
\kappa \equiv \frac{\partial}{\partial \tilde{Z}}+\lambda_{1} \eta \frac{\partial}{\partial \xi}-\frac{\partial^{2}}{\partial \eta^{2}}
$$


First from (4 2) we obtain

$$
v^{v-1}=-\imath \bar{A}(\mathrm{X}, \tilde{T}, \hat{Z}) \epsilon^{\imath\urcorner \varepsilon} g_{1}+\mathrm{c} \mathrm{c}
$$

where we have defined

$$
A(X, T, Z)=A\left(X^{-}, \mu_{0}^{-1} \tilde{T}+\tilde{Z}, \mu_{0} \tilde{Z}\right)=\bar{A}\left(X^{-}, \grave{T} . \dot{Z}\right),
$$

and $g_{1}$ is a constant. Matching with the outer solution ( $\left.33 \mathrm{~b}\right),(36 \mathrm{a})$ shows that

$$
g_{1}=B^{+}=B^{-}
$$

If we put $\tilde{V}_{-1}=-\imath \vec{A}(X, \tilde{T}, \tilde{Z}) g_{1}, \quad w_{0}=\tilde{W}_{0} e^{\imath\urcorner \xi}+c c, \quad u_{0}=\tilde{U}_{0} e^{\imath \gamma \xi}+$ c.c., $\quad v_{0}=$ $\tilde{V}_{0} e^{2 \gamma \xi}+$ c.c., then from (43) using (4 10), we find that

$$
\tilde{W}_{0}=d_{0} \nu \int_{0}^{\infty} \bar{A}(X, \tilde{T}, \tilde{Z}-s) e^{-\sigma s^{3}-\imath \nu s \eta} d \eta
$$

where

$$
\nu=\lambda_{1} \gamma, \quad \sigma=\frac{\nu^{2}}{3}, \quad d_{0}=\frac{2 g_{1} \mu_{1}}{\lambda_{1} \gamma}
$$

Also

$$
\begin{gathered}
\tilde{U}_{0}=-\frac{1}{\imath \gamma} \tilde{W}_{0 Z}+\tilde{U}_{00}(\tilde{T}, \hat{Z}), \\
\tilde{V}_{0}=-\imath \gamma \eta \tilde{U}_{00}+\tilde{V}_{00}(\tilde{T}, \tilde{Z}),
\end{gathered}
$$

where $\tilde{U}_{00}, \tilde{V}_{00}$ may be determined through matching In fact from (3.1), (3.3), (3.6), (4.14b) we see that

$$
A C^{+}=A C^{-}=\gamma \tilde{U}_{00}
$$

We next consider the solutions of the second orde1 problem (4.5). The forcing terms in the equation for $W_{1}$ indicate a solution of the form

$$
W_{1}=\tilde{W}_{10}+\tilde{W}_{12} e^{2 \gamma \gamma}+c c
$$

where $\tilde{W}_{10}, \tilde{W}_{12}$ are independent of $\xi$. From (45c) the equation for $\tilde{W}_{10}$ is

$$
\mathcal{L}_{0} \tilde{W}_{10}=-\tilde{V}_{-1}^{(c)} \frac{\partial \tilde{W}_{0}}{\partial \eta}
$$

where the operator $\mathcal{L}_{n}$ is defined by

$$
\mathcal{L}_{n}=\frac{\partial}{\partial \tilde{Z}}+2 \imath n \gamma \eta-\frac{\partial^{2}}{\partial \eta^{2}}
$$

The equation for $\tilde{W}_{10}$ may be solved to give

$$
\tilde{W}_{10}=-g_{1}^{(c)} d_{0} \nu^{2} \int_{0}^{\infty} d s \int_{0}^{\infty} \bar{A}^{(c)}\left(X, \tilde{T}, \tilde{Z}-s_{1}\right) \bar{A}\left(X, \tilde{T}, \tilde{Z}-s-s_{1}\right) s K_{4}^{(0)}\left(s, s_{1} \mid \sigma\right) e^{-i \nu \eta s} d s_{1}
$$

where the notation $A^{(c)}$ denotes the complex conjugate of $A$ and

$$
K_{4}^{(0)}\left(s_{1}, s_{2} \mid \sigma\right)=e^{-\sigma\left(s_{1}^{3}+3 s_{1}^{2} s_{2}\right)}
$$


The equation for $\tilde{T}_{12}$ is

$$
\mathcal{L}_{2} \dot{I I}_{12}^{r}=-\tilde{V}_{-1} \dot{I I}_{\text {on }}
$$

This is solved to obtain

$$
\tilde{W}_{12}=d_{1} \int_{0}^{\infty} d s_{1} \int_{0}^{\infty} s_{1} \bar{A}\left(X . \tilde{T}, \tilde{Z}-s_{1}\right) \bar{A}\left(X, \tilde{T}, \tilde{Z}-s_{1}-s_{2}\right) K_{4}^{-(1)}\left(s_{1} . s_{2}\right) e^{-\imath \nu \eta\left(2 s_{1}+s_{2}\right)} d s_{2}
$$

where

$$
K_{4}^{-(1)}\left(s_{1}, s_{2}\right)=e^{-\sigma\left(s_{1}^{3}+3 s_{1}^{2} s_{2}+6 s_{2}^{2}+4 s_{2}^{3}\right)}
$$

and

$$
d_{1}=\imath g_{1}^{2} \mu_{1} \lambda_{1} \gamma
$$

Next if we differentiate (4.5b) with respect to $\eta$ and use the continuity equation (4.5a) this gives

$$
\mathcal{K} \Omega_{1}=\lambda_{1} w_{1 Z}-v_{-1} \Omega_{0 \eta}
$$

and we have defined $\Omega_{n}=\frac{\partial U_{n}}{\partial \eta}$. The right hand side of (4.20) suggests that

$$
\Omega_{1}=\tilde{\Omega}_{10}+\tilde{\Omega}_{12} e^{2 \imath\urcorner \xi}+\text { c.c }
$$

The mean flow component of $\Omega_{1}, \tilde{\Omega}_{10}$ satisfies the equation

$$
\mathcal{L}_{0} \tilde{\Omega}_{10}=-\tilde{V}_{-1}^{(c)} \frac{\partial \tilde{\Omega}_{0}}{\partial \eta}+\lambda_{1}\left(\mu_{0}^{-1} \frac{\partial}{\partial \tilde{Z}}-\frac{\partial}{\partial \tilde{T}}\right) \hat{V}_{10}
$$

Using (4 14a). (4.18) it is found that $\tilde{\Omega}_{10}$ is given by

$$
\begin{gathered}
\tilde{\Omega}_{10}=-g_{1}^{(c)} \frac{d_{0}}{\gamma} \nu^{3} \int_{0}^{\infty} d s \int_{0}^{\infty} s^{2} \bar{A}^{(c)}\left(X, \hat{T}, \tilde{Z}-s_{2}\right) K_{4}^{-(0)}\left(s, s_{2}\right) \times \\
e^{-\imath \nu s \eta}\left(\mu_{0}^{-1} \frac{\partial}{\partial \tilde{Z}}-\frac{\partial}{\partial \tilde{T}}\right) \bar{A}\left(X, \tilde{T}, \hat{Z}-s_{2}-s\right) d s_{2} \\
-g_{1}^{(c)} \lambda_{1} d_{0} \nu^{2} \int_{0}^{\infty} d s \int_{0}^{\infty} d s_{2} \int_{0}^{\infty} s K_{4}^{-(0)}\left(s, s_{1}+s_{2} \mid \sigma\right) e^{-\imath \nu \eta s} \times \\
\left(\mu_{0}^{-1} \frac{\partial}{\partial \hat{Z}}-\frac{\partial}{\partial \tilde{T}}\right)\left[\bar{A}^{(c)}\left(\tilde{X}, \tilde{T}, \hat{Z}-s_{2}-s_{1}\right) \bar{A}\left(X, \tilde{T} \cdot \tilde{Z}-s_{2}-s_{1}-s\right)\right] d s_{1}
\end{gathered}
$$

The $e^{2 v \gamma \xi}$ component of $\Omega_{1}$ satisfies the equation

$$
\mathcal{L}_{2} \tilde{\Omega}_{12}=-\tilde{V}_{-1} \tilde{\Omega}_{0 \eta}+\lambda_{1} \tilde{W}_{12 Z}
$$

Thus using the solutions for $\Omega_{0}, \tilde{W}_{12}$, it is found that

$$
\begin{gathered}
\tilde{\Omega}_{12}=-2 d_{2} \nu^{4} \int_{0}^{\infty} d s \int_{0}^{\infty} s^{2} K_{4}^{r(1)}\left(s, s_{1}\right) e^{-\imath \eta_{\nu}\left(2 s_{1}+s\right)} \times \\
\bar{A}\left(X, T, \tilde{Z}-s_{1}\right) \frac{\partial}{\partial Z}\left(\bar{A}\left(X, \tilde{T}, \bar{Z}-s_{1}-s\right)\right) d s_{1} \\
+4 d_{3} \nu^{4} \int_{0}^{\infty} d s \int_{0}^{\infty} d s_{1} \int_{0}^{\infty} s_{1} K_{4}^{(1)}\left(s_{1}, s+s_{2}\right) e^{-\imath \eta \nu\left(s_{1}+2 s+2 s_{2}\right)} \times
\end{gathered}
$$




$$
\frac{\partial}{\partial Z}\left[\bar{A}\left(X, \tilde{T} \cdot \tilde{Z}-s-s_{2}\right) \bar{A}\left(X, T . \dot{Z}-s_{2}-s_{1}-s\right)\right] d s_{2},
$$

where the constants are defined by

$$
d_{2}=-\frac{g_{1} d_{0}}{2 \nu \gamma}, \quad d_{3}=\frac{d_{0} g_{1}}{4 \nu \gamma}
$$

We turn our attention next to the third order problem defined by (4 6a-d) which determines the nonlinear jump. It is only necessary to calculate the $e^{2 \uparrow \xi}$ components of $\mathrm{IT}_{2}$ and $U_{2}, \tilde{W}_{21}$ and $\tilde{U}_{21}$ respectively From $(4.6 \mathrm{c})$ is is seen that

$$
\mathcal{L}_{1} \tilde{W}_{21}=R_{1}+R_{2}+R_{3}
$$

where

$$
\begin{gathered}
R_{1}=-\eta\left[\lambda_{1} \tilde{W}_{0 X}+2 \mu_{2} \tilde{V}_{-1}+\mu_{1} \tilde{W}_{0 z}-\imath \gamma \mu_{1} \tilde{U}_{00}\right] \\
R_{2}=-\tilde{p}_{0 Z}-\mu_{1} \tilde{V}_{00}-V_{B}^{(0)} \tilde{W}_{0 \eta}+\mu_{0} Y_{c z} \tilde{W}_{0 \eta}
\end{gathered}
$$

and

$$
R_{3}=-\left[\tilde{V}_{-1}\left[\tilde{W}_{12 \eta}+\tilde{V}_{-1}\left(\tilde{W}_{10 \eta}+\tilde{W}_{0}^{(c)} \eta\right)\right]\right.
$$

Writıng

$$
\tilde{W}_{21}=\tilde{W}_{21}^{(1)}+\tilde{W}_{21}^{(2)}+\tilde{W}_{21}^{(3)}
$$

with $\mathcal{L}_{1} \tilde{W}_{21}=R_{3}$, then it can be shown that

$$
\begin{aligned}
\tilde{W}_{21}^{(1)}= & \frac{1}{\imath \gamma}\left[\eta \tilde{W}_{0 \eta X}-\frac{2}{3 \imath \nu} \tilde{W}_{0 \eta \eta \eta X}\right]+\frac{\mu_{1}}{i \nu} \frac{\partial}{\partial Z}\left[\eta \left[\tilde{W}_{0 \eta}-\frac{2}{3 \nu \nu}\left[\tilde{W}_{0 \eta \eta \eta}\right]\right.\right. \\
& +\frac{2 \mu_{2}}{\mu_{1}}\left[\eta \tilde{W}_{0}-\frac{1}{\imath \nu} \tilde{W}_{0 \eta \eta}\right]+\imath \gamma \mu_{1}\left[\eta F_{0}-\frac{1}{\imath \nu} F_{0 \eta n}\right]
\end{aligned}
$$

where

$$
F_{0}=\int_{0}^{\infty} \tilde{U}_{00}(X, \tilde{T}, \tilde{Z}-s) e^{-\sigma s^{3}-\imath \nu \eta s} d s .
$$

Similarly the solution to $\mathcal{L}_{1} \tilde{W}_{21}^{(2)}=R_{2}$ is given by

$$
\tilde{W}_{21}^{(2)}=-\frac{\lambda}{2 \gamma \mu_{1}} \tilde{W}_{0 Z}-\frac{\mu_{1}}{\mu_{0}} F_{1}+\frac{1}{2 i \nu}\left(V_{B 0}^{(0)}-\mu_{0}{ }^{\circ} c z\right) \tilde{I}_{0 \eta \eta}^{r},
$$

where

$$
F_{1}=\int_{0}^{\infty} \tilde{V}_{00}(X, \tilde{T}, \tilde{Z}-s) e^{-\sigma s^{3}-\imath \nu \eta s} d s
$$

Next, using (4 9), (4.16), (4 18), the solution to $\mathcal{L}_{1} \tilde{W}_{21}^{(3)}=R_{3}$ is found to be

$$
\begin{gathered}
\tilde{W}_{21}^{(3)}=-d_{4} \nu^{3} \int_{0}^{\infty} d s_{2} \int_{0}^{\infty} d s_{1} \int_{s_{2}}^{\infty} s_{2}^{2} \bar{A}\left(X, \tilde{T}, \tilde{Z}-s+s_{2}\right) \bar{A}^{(c)}\left(X^{-} . \tilde{T} . \tilde{Z}-s+s_{2}-s_{1}\right) \times \\
\bar{A}\left(X, \tilde{T}, \tilde{Z}-s-s_{1}\right) e^{-i \nu s \eta} K_{4}^{(2)}\left(s, s_{1} . s_{2}\right) d s \\
\quad+d_{5} \nu^{3} \int_{0}^{\infty} d s_{2} \int_{0}^{\infty} d s_{1} \int_{-s_{2}}^{\infty} s_{2}^{2} \bar{A}\left(X^{-}, \tilde{T}, \tilde{Z}-s-s_{2}\right) \times \\
\bar{A}\left(X, \tilde{T}, \tilde{Z}-s-s_{2}-s_{1}\right) \bar{A}^{(c)}\left(\mathcal{X}, \tilde{T}, \tilde{Z}-s-2 s_{2}-s_{1}\right) \epsilon^{-i \nu s \eta} K_{4}^{(3)}\left(s, s_{1}, s_{2}\right) d s
\end{gathered}
$$




$$
\begin{gathered}
-\bar{d}_{6} \nu^{4} \int_{0}^{\infty} d s_{2} \int_{s_{2}}^{x} d s \int_{0}^{s_{2}} s_{2} s_{1} \bar{A}^{(c)}\left(X . \dot{T} \dot{Z}-\cdot+s_{2}\right) \times \\
\bar{A}\left(X, \tilde{T}, \tilde{Z}-\frac{1}{2}\left(2 s-s_{2}-s_{1}\right)\right) \bar{A}\left(X . \tilde{T}, \tilde{Z}-\frac{1}{2}\left(2 s+s_{1}-s_{2}\right) L_{4}^{r(5)}\left(s_{.} s_{1} . s_{2}\right) \epsilon^{-\imath \nu \eta s} d s_{1},\right.
\end{gathered}
$$

where

$$
\begin{array}{r}
K_{4}^{(2)}\left(s, s_{1}, s_{2}\right)=e^{-\sigma\left(3 s_{1} s_{2}^{2}+s^{2}\right)} \\
K_{4}^{(3)}\left(s, s_{1}, s_{2}\right)=e^{-\sigma\left(2 s_{2}^{3}+3 s_{2}^{2} s_{1}+s^{3}\right)} \\
K_{4}^{-(4)}\left(s, s_{1}, s_{2}\right)=e^{-\sigma\left(s^{2}+\frac{\dot{z}_{1}^{2}-s_{2}^{3}}{2}\right)} \\
d_{4}=\left|g_{1}\right|^{2} d_{0}, \quad d_{5}=g_{1}^{2} d_{0}^{(c)}, \quad \bar{d}_{6}=\frac{\left|g_{1}\right|^{2} d_{0}}{2 \nu} .
\end{array}
$$

Finally, one other quantity which is required for the calculation of the jump is $\tilde{\Omega}_{21}$, the $e^{\imath \gamma \xi}$ component of $\tilde{\Omega}_{2}$ which satisfies

$$
\mathcal{L}_{1} \tilde{\Omega}_{21}=Q_{1}+Q_{2}+Q_{3}+Q_{4}
$$

with

$$
\begin{aligned}
& Q_{1}=-\eta\left(\lambda_{1} \tilde{\Omega}_{0 X}+6 \lambda_{3} \tilde{V}_{-1}+\mu_{1} \tilde{\Omega}_{0 Z}+\gamma^{2} \lambda_{1} \tilde{V}_{-1}\right) \\
& Q_{2}=-\mu_{1} \tilde{u}_{0 Z}+2 \gamma \tilde{V}_{-1 \tilde{Z}}-V_{B 0}^{(0)} \tilde{\Omega}_{0 \eta}+\lambda_{1} I_{c z} \tilde{V}_{0 \eta} . \\
& Q_{3}=-\tilde{V}_{-1}\left(\tilde{\Omega}_{10 \eta}+\tilde{\Omega}_{10}^{(c)} \eta\right)-\tilde{V}_{-1}^{(c)} \Omega_{12 \eta} . \\
& Q_{4}=\lambda_{1} \tilde{W}_{21 Z} .
\end{aligned}
$$

If we write $\tilde{\Omega}_{21}=\tilde{\Omega}_{21}^{(1)}+\tilde{\Omega}_{21}^{(2)}+\tilde{\Omega}_{21}^{(3)}+\tilde{\Omega}_{21}^{(4)}$ with $\mathcal{L}_{1} \tilde{\Omega}_{21}^{(g)}=Q$, then it is easy to show that

$$
\begin{gathered}
\tilde{\Omega}_{21}^{(1)}=\left(\frac{6 \lambda_{3}+\gamma^{2} \lambda_{1}}{\mu_{1}}\right)\left(\eta \tilde{W}_{0}-\frac{\tilde{W}_{0 \eta n}}{\imath \mu}\right)+\frac{1}{2 \imath \gamma^{2}} \frac{\partial}{\partial Z}\left(\eta W_{0 \eta \eta}-\frac{1}{2 i \nu} W_{0 \eta \eta \eta \eta}\right) \\
+\frac{\mu_{1}}{2 \gamma \nu} \frac{\partial^{2}}{\partial Z^{2}}\left(\eta W_{0 \eta \eta}-\frac{1}{2 \imath \nu} W_{0 \eta \eta \eta \eta}\right) .
\end{gathered}
$$

The solution to $\mathcal{L}_{1} \tilde{\Omega}_{21}^{(2)}=Q_{2}$ is using $(4.3 \mathrm{c}),(4.12),(4.14) .(4.26)$,

$$
\tilde{\Omega}_{21}^{(2)}=\frac{\mu_{1}}{\gamma \nu} \frac{\partial^{2}}{\partial Z^{2}}\left(\tilde{W}_{0 \eta}\right)-\mu_{1} \frac{\partial F_{0}}{\partial Z}-\frac{\imath \gamma}{\mu_{1}} \frac{\partial \tilde{W}_{0}}{\partial \tilde{Z}}+\frac{V_{B 0}^{(0)}}{3 \imath \nu} \grave{\varrho}_{0 \eta \eta}-\frac{\lambda_{1} \Psi_{c z}}{2_{\imath \nu}} \tilde{W}_{0 \eta \eta}
$$

$\operatorname{Next} \tilde{\Omega}_{21}^{(3)}$ satisfies

$$
\mathcal{L}_{1} \tilde{\Omega}_{21}^{(3)}=Q_{3}^{(1)}+Q_{3}^{(2)}+Q_{3}^{(3)}
$$

where

$$
Q_{3}^{(1)}=-\tilde{V}_{-1} \tilde{\Omega}_{10 \eta}, \quad Q_{3}^{(2)}=-\tilde{V}_{-1} \tilde{\Omega}_{10 \eta}^{(c)}, \quad Q_{3}^{(3)}=-\tilde{I}_{-1}^{(c)} \Omega_{12 \eta}
$$

The solution $\tilde{\Omega}_{21}^{(3)}$ is decomposed into

$$
\tilde{\Omega}_{21}^{(3)}=\sum_{\jmath=1}^{3} \tilde{\Omega}_{21}^{(3, \jmath)}
$$


with $\mathcal{L}_{1} \hat{\Omega}_{21}^{(3, \jmath)}=Q_{3}^{(\jmath)}$, and each of the $\dot{\Omega}_{21}^{(3 \jmath)}$ components are solved for separately Howerer only the $\hat{\Omega}_{21}^{(3,2)}$ term provides a non-zeio contisbution to the amplitude equation ${ }_{\text {sing }}$ $(4.22)$, it is found that

$$
\begin{gathered}
\tilde{\Omega}_{21}^{(3,2)}=-d_{6} \nu^{4} \int_{0}^{\infty} d s_{3} \int_{0}^{\infty} d s_{2} \int_{-s_{3}}^{\infty} s_{3}^{3} \bar{A}\left(\dot{X} . \dot{T}, \dot{Z}-s-s_{3}\right) \times \\
\bar{A}\left(X, \tilde{T}, \tilde{Z}-s_{2}-s-s_{3}\right) K_{4}^{r(3)}\left(s, s_{2}, s_{3}\right) e^{-\imath \nu \eta s} \frac{\partial}{\partial Z}\left[\bar{A}^{(c)}\left(\mathcal{X}, \dot{T} . \dot{Z}-s_{2}-s-2 s_{3}\right)\right] d s \\
+d_{\tau} \nu^{3} \int_{0}^{\infty} d s_{3} \int_{0}^{\infty} d s_{2} \int_{0}^{\infty} d s_{1} \int_{-s_{3}}^{\infty} s_{3}^{2} \bar{A}\left(X, \tilde{T}, \tilde{Z}-s-s_{3}\right) K_{4}^{-(3)}\left(s, s_{2}+s_{1}, s_{3}\right) \times \\
e^{-\imath \nu \eta s} \frac{\partial}{\partial Z}\left[\bar{A}\left(X, \tilde{T}, \tilde{Z}-s-s_{3}-s_{2}-s_{1}\right) \bar{A}^{(c)}\left(X, \tilde{T}, \tilde{Z}-s_{2}-s_{1}-s-2 s_{3}\right)\right] d s,
\end{gathered}
$$

where

$$
d_{6}=-\frac{g_{1}^{2} d_{0}^{(c)}}{\gamma}, \quad d_{7}=g_{1}^{2} d_{0}^{(c)} \lambda_{1} .
$$

Finally the equation for $\tilde{\Omega}_{21}^{4}$ is

$$
\mathcal{L}_{1} \tilde{\Omega}_{21}^{(4)}=\lambda_{1} \tilde{W}_{21 Z}=\sum_{\jmath=1}^{3} \lambda_{1} \tilde{W}_{21 Z}^{(\jmath)}
$$

Writing $\tilde{\Omega}_{21}^{(4)}=\sum_{\jmath=1}^{3} \tilde{\Omega}_{21}^{(4,3)}$ where

$$
\mathcal{L}_{1} \tilde{\Omega}_{21}^{(4, \jmath)}=\lambda_{1} \tilde{W}_{21 Z}^{(\jmath)}
$$

from $(4.26),(4.29 a)$ we obtain

$$
\begin{aligned}
& \tilde{\Omega}_{21}^{(4,1)}=+\frac{1}{2 \gamma^{2}} \frac{\partial^{2}}{\partial Z \partial X}\left[\eta \tilde{W}_{0 \eta \eta}-\frac{1}{2 \imath \nu} \tilde{W}_{0 \eta \eta \eta \eta}^{\prime}\right]-\frac{1}{6 \imath \gamma^{2} \nu} \frac{\partial^{2}}{\partial Z \partial X} W_{0 \eta \eta \eta \eta} \\
&+ \frac{\mu_{1}}{2 \gamma^{2} \lambda_{1}} \frac{\partial^{2}}{\partial Z^{2}}\left[\eta W_{0 \eta \eta}-\frac{1}{2 \imath \nu} W_{0 \eta \eta \eta \eta}\right]-\frac{\mu_{1}}{6 \imath \gamma \nu^{2}} \frac{\partial^{2}}{\partial Z^{2}}\left(W_{0 \eta \eta \eta \eta}^{r}\right) \\
&-\frac{2 \mu_{2} \lambda_{1}}{\mu_{1}}\left[-\frac{1}{\imath \nu} \frac{\partial}{\partial Z}\left(\eta W_{0 \eta}-\frac{2}{3 \imath \nu} W_{0 \eta \eta \eta}\right)+\frac{1}{3 \nu^{2}} \frac{\partial}{\partial Z} W_{0 \eta \eta \eta}\right] \\
&-\mu_{1}\left[\frac{\partial}{\partial Z}\left(\eta F_{0 \eta}-\frac{2}{3 \imath \nu} F_{0 \eta \eta \eta}\right]+\frac{1}{3 \nu \imath} \frac{\partial}{\partial Z}\left(F_{0 \eta \eta \eta}\right)\right],
\end{aligned}
$$

and

$$
\tilde{\Omega}_{21}^{(4,2)}=-\frac{1}{\gamma^{2} \mu_{1}} \frac{\partial^{2}}{\partial Z^{2}} \tilde{W}_{0 \eta}+\frac{1}{2 \gamma \mu_{0}} \frac{\partial}{\partial Z} F_{1 \eta}+\frac{1}{6 \gamma \nu}\left(V_{B 0}^{(0)}-\mu_{0} I_{c z}\right) \frac{\partial}{\partial Z}\left(\tilde{W}_{0 \eta \eta \eta}\right) .
$$

In (4.28) the solution for $\tilde{W}_{21}^{(3)}$ inrolves three triple integrals and only the second triple integral generates a term in the solution for $\tilde{\Omega}_{21}^{(4,3)}$ which contributes to the amplitude equation The solution to (433) with just this term present is

$$
\tilde{\Omega}_{21}^{(4,3)}=\lambda_{1} d_{5} \nu^{3} \int_{0}^{\infty} d s_{2} \int_{0}^{\infty} d s \int_{-s_{2}}^{\infty}\left(s_{2}+s\right) s_{2}^{2} L_{4}^{-(3)}\left(s_{.} s_{1}, s_{2}\right) e^{-i \nu s \eta} \times
$$

$$
\frac{\partial}{\partial Z}\left[\bar{A}\left(X, \tilde{T}, \tilde{Z}-s-s_{2}\right) \bar{A}\left(X, \tilde{T}, \tilde{Z}-s-s_{2}-s_{1}\right) \bar{A}^{(c)}\left(\mathcal{X}^{-}, \tilde{T} . \dot{Z}-s-2 s_{2}-s_{1}\right)\right] d s .
$$

We turn our attention next to the calculation of jumps in (3.10) 


\section{Calculation of the jumps and the amplitude equa- tion}

From (3.1), (39), (4 Ib), (4.11), (4.14), (4.16) it is clear that $\left(\tilde{V}_{1}\right)_{z_{c}}^{\xi_{c}+}=0$ In addition these equations show that

$$
\left(\tilde{V}_{1 \nu^{\prime}}\right)_{\zeta_{c}^{c}}^{c_{c}^{+}}=\left(g_{+}-g_{-}\right)=-2 \gamma \int_{\eta=-\infty}^{\infty \infty}\left(\dot{\Omega}_{21}\right) d \eta
$$

where the notation $\int^{*}$ denotes the finite part of the integral The integral in (5.1) is now evaluated using the solution for $\tilde{\Omega}_{21}$ obtained in the pievious section. First from (4.30) we find that

$$
\int_{-\infty}^{* \infty} \tilde{\Omega}_{21}^{(1)} d \eta=\frac{d_{0}}{\mu_{1} \nu}\left(6 \lambda_{3}+\gamma^{2} \lambda_{1}\right) s_{n} \pi \imath \frac{\partial}{\partial \hat{Z}} \bar{A}(\lambda, \hat{T}, \tilde{Z}),
$$

where $s_{n}=\operatorname{sgn}\left(\lambda_{1}\right)$, and only the first term in (4.30) gives a nonzero contribution.

Next using (4 31) we have

$$
\int_{-\infty}^{* \infty} \tilde{\Omega}_{21}^{(2)} d \eta=-s_{n} \frac{\mu_{1} \pi}{\nu} \frac{\partial U_{00}}{\partial Z}(X, \tilde{T}, \tilde{Z})-s_{n} \frac{d_{0} \pi \imath \gamma}{\mu_{1}} \frac{\partial}{\partial \tilde{Z}} \bar{A}(X, \tilde{T}, \tilde{Z}) .
$$

Whereas $(5.2 \mathrm{a}, \mathrm{b})$ are linear contrıbutions, the $\tilde{\Omega}_{21}^{(3,2)}$ terms gives a nonlinear jump From (4.32) we find that

$$
\begin{gathered}
\int_{-\infty}^{* \infty} \tilde{\Omega}_{21}^{(3,2)} d \eta=-s_{n} d_{6} \nu^{3} 2 \pi \int_{0}^{\infty} d s_{3} \int_{0}^{\infty} s_{3}^{3} \bar{A}\left(X, \tilde{T}, \tilde{Z}-s_{3}\right) \times \\
\bar{A}\left(X, \tilde{T}, \tilde{Z}-s_{2}-s_{3}\right){K_{4}^{-}}_{4}^{(3)}\left(0, s_{2}, s_{3}\right) \frac{\partial}{\partial Z}\left[\bar{A}^{(c)}\left(X, \tilde{T} \cdot \tilde{Z}-s_{2}-2 s_{3}\right)\right] d s_{2} \\
+2 s_{n} \pi d_{7} \nu^{2} \int_{0}^{\infty} d s_{3} \int_{0}^{\infty} d s_{2} \int_{0}^{\infty} s_{3}^{2} \bar{A}\left(X, \tilde{T}, \tilde{Z}-s_{3}\right) \cdot K_{4}^{(3)}\left(0, s_{2}+s_{1}, s_{3}\right) \\
\frac{\partial}{\partial Z}\left[\bar{A}\left(X, \tilde{T}, \tilde{Z}-s_{3}-s_{2}-s_{1}\right) \bar{A}^{(c)}\left(X, \tilde{T}, \tilde{Z}-s_{2}-s_{1}-2 s_{3}\right)\right] d s_{1} .
\end{gathered}
$$

Next the contribution from the $\tilde{\Omega}_{21}^{(4,1)}$ term is

$$
\int_{-\infty}^{* \infty} \tilde{\Omega}_{21}^{(4,1)} d \eta=s_{n} \frac{2 \mu_{2} d_{0} \pi}{2 \gamma \mu_{1}} \frac{\partial}{\partial Z} \bar{A}(X, \tilde{T}, \tilde{Z})+s_{n} \frac{\mu_{1} \pi}{\nu} \frac{\partial}{\partial Z} U_{00}(\mathcal{X}, \tilde{T}, \tilde{Z}) .
$$

The $\tilde{\Omega}_{21}^{(4,2)}$ term gives zero contribution. The $\tilde{\Omega}_{21}^{(4,3)}$ term gives a nonlinear jump term

$$
\begin{array}{r}
\int_{-\infty}^{* \infty} \tilde{\Omega_{21}}(4,3) d \eta=s_{n} \lambda_{1} d_{5} \nu^{2} 2 \pi \int_{0}^{\infty} d s_{2} \int_{0}^{\infty} d s_{1} s_{2}^{3} K_{4}^{(3)}\left(0, s_{1}, s_{2}\right) \times \\
\frac{\partial}{\partial Z}\left[\bar{A}\left(X, \tilde{T}, \tilde{Z}-s_{2}\right) \bar{A}\left(X, \tilde{T}, \tilde{Z}-s_{2}-s_{1}\right) \bar{A}^{(c)}\left(X, \tilde{T} . \tilde{Z}-2 s_{2}-s_{1}\right)\right]
\end{array}
$$

Hence finally collecting all the terms (5 2a-e), restoring the variables $X, Z, T$, and substituting into the (3.10) gives the resulting amplitude equation as 


$$
\begin{gathered}
\frac{I_{1}}{\gamma} \frac{\partial A}{\partial T}+\frac{I_{2}}{\gamma} \frac{\partial A}{\partial Z}+\gamma I_{3} \frac{\partial A}{\partial X}=\left[i_{01} i_{1}\right]_{\mathrm{Y}=0} \\
+\imath s_{n} \gamma g_{1}\left\{\frac{g_{1} \pi}{\lambda_{1} \gamma^{2}}\left(2 \mu_{2}-\frac{6 \lambda_{3} \mu_{0}}{\lambda_{1}}\right) \frac{\partial A}{\partial Z}-\frac{g_{1} \pi 6 \lambda_{3}}{\lambda_{1}^{2}{ }_{1}^{2}} \frac{\partial A}{\partial T}\right\} \\
+2 s_{n} \pi \gamma^{2} g_{1}^{2}\left|g_{1}\right|^{2} \mu_{1} \lambda_{1}^{2}\left[+\int_{0}^{\infty} d s_{3} \int_{0}^{\infty} d s_{2} s_{3}^{3} A\left(X, T-s_{3}, Z-\mu_{0} s_{3}\right) K_{4}^{(3)}\left(0, s_{2}, s_{3}\right) \times\right. \\
A\left(X, T-s_{2}-s_{3}, Z-\mu_{0}\left(s_{2}+s_{3}\right)\right) \frac{\partial}{\partial Z} A^{(c)}\left(X, T-s_{2}-2 s_{3}, Z-\mu_{0}\left(s_{2}+2 s_{3}\right)\right) \\
+\int_{0}^{\infty} d s_{3} \int_{0}^{\infty} d s_{2} \int_{0}^{\infty} d s_{1} s_{3}^{2} A\left(X, T-s_{3}, Z-\mu_{0} s_{3}\right) K_{4}^{(3)}\left(0, s_{2}+s_{1}, s_{3}\right) \times \\
\frac{\partial}{\partial Z}\left[A \left(X, T-s_{3}-s_{2}-s_{1}, Z-\mu_{0}\left(s_{3}+s_{2}+s_{1}\right) A^{(c)}\left(X, T-s_{2}-s_{1}-2 s_{3}, Z-\mu_{0}\left(s_{2}+s_{1}+2 s_{3}\right)\right]\right.\right. \\
+\int_{0}^{\infty} d s_{2} \int_{0}^{\infty} d s_{1} s_{2}^{3} K_{4}^{(3)}\left(0, s_{1}, s_{2}\right) \frac{\partial}{\partial Z}\left[A\left(X, T-s_{2} . Z-\mu_{0} s_{2}\right) \times\right. \\
\left.A\left(X, T-s_{2}-s_{1}, Z-\mu_{0}\left(s_{2}+s_{1}\right)\right) A^{(c)}\left(X, T-2 s_{2}-s_{1}, Z-\mu_{0}\left(2 s_{2}+s_{1}\right)\right)\right]
\end{gathered}
$$

This can be written in a more compact form as

$$
\frac{\bar{I}_{1}}{\gamma} \frac{\partial A}{\partial T}+\frac{\bar{I}_{2}}{\gamma} \frac{\partial A}{\partial Z}+\gamma I_{3} \frac{\partial A}{\partial X}=\left[\tilde{V}_{0 Y}, \tilde{V}_{1}\right]_{Y=0}+M\left(J_{1}+J_{2}+J_{3}\right)
$$

where

$$
\begin{gathered}
M=2 s_{n} \pi \gamma^{2} g_{1}^{2}\left|g_{1}^{2}\right| \mu_{1} \lambda_{1}^{2}, \quad \bar{I}_{1}=\int_{C} \frac{U_{B Y Y}}{U_{B}^{2}} \tilde{V}_{0}^{2} d Y, \quad \bar{I}_{2}=-\int_{C}\left(\frac{I_{B I Y}}{l_{B}^{-}}-\frac{W_{B} U_{B Y Y}}{U_{B}^{2}}\right) \tilde{V}_{0}^{2} d Y, \\
J_{1}=\int_{0}^{\infty} d s_{3} \int_{0}^{\infty} d s_{2} s_{3}^{3} A\left(X, T-s_{3}, Z-\mu_{0} s_{3}\right) K_{4}^{(0)}\left(s_{2}, s_{3} \mid \sigma\right) \times \\
A\left(X, T-s_{2}-s_{3}, Z-\mu_{0}\left(s_{2}+s_{3}\right)\right) \frac{\partial}{\partial Z} A^{(c)}\left(X, T-s_{2}-2 s_{3} . Z-\mu_{0}\left(s_{2}+2 s_{3}\right)\right), \\
J_{2}=\int_{0}^{\infty} d s_{3} \int_{0}^{\infty} d s_{2} s_{3}^{2} s_{2} A\left(X, T-s_{3}, Z-\mu_{0} s_{3}\right) K_{4}^{-(0)}\left(s_{2}, s_{3} \mid \sigma\right) \times \\
\frac{\partial}{\partial Z}\left[A\left(X, T-s_{3}-s_{2}, Z-\mu_{0}\left(s_{3}+s_{2}\right)\right) A^{(c)}\left(X, T-s_{2}-2 s_{3} . Z-\mu_{0}\left(s_{2}+2 s_{3}\right)\right],\right. \\
J_{3}=\int_{0}^{\infty} d s_{2} \int_{0}^{\infty} d s_{1} s_{2}^{3} K_{4}^{(0)}\left(s_{1}, s_{2} \mid \sigma\right) \frac{\partial}{\partial Z}\left[A\left(X . T-s_{2} . Z-\mu_{0} s_{2}\right) \times\right. \\
\left.A\left(X, T-s_{2}-s_{1}, Z-\mu_{0}\left(s_{2}+s_{1}\right)\right) A^{(c)}\left(X, T-2 s_{2}-s_{1}, Z-\mu_{0}\left(2 s_{2}+s_{1}\right)\right)\right],
\end{gathered}
$$

and the path of integration $C$ for $\bar{I}_{1}, \bar{I}_{2}$ is along the positive real axis with an indentation below/above the point $Y=Y_{c}$ depending on whether $\lambda_{1}$ is positive/negative.

Equation (5 4) is the main result of this paper. A generalisation of this equation for the flow over a rotating disk is given in Appendix A. In the next section we consider a few of the properties of this equation and the numerical solution of a special case. 


\section{Special cases and results}

The solution of the full equation (5.4) is in general quite difficult because of the coupling with the wall layer and secondly because of the form of the nonlinearity. There are however some special cases which can be considered further Below we have used the normalisation $\tilde{V}_{0 Y}(0)=1$ for the solution of the leading order eigenvalue problem (34).

(a) If we consider the plane wave $A=\tilde{A} e^{2\left(\alpha_{1} X+\beta_{1} Z\right)-\imath w_{1} T}$ with $a_{1} \cdot \beta_{1}, \omega_{1}$ all real, then the contribution from the nonlinear terms $J_{1}+J_{2}+J_{3}$ is identically zero and (5.4) leads to the linear dispersion relation for neutral waves as

$$
-\frac{\imath \bar{I}_{1} \omega_{1}}{\gamma}+\frac{\imath \bar{I}_{2} \beta_{1}}{\gamma}+\imath \gamma I_{3} \alpha_{1}=-\frac{\imath e^{\imath \frac{\overline{6}}{\overline{6}} s_{n}}}{\left(\gamma\left|\bar{\lambda}_{1}\right|\right)^{\frac{3}{3}}} G\left(\xi_{0}\right)
$$

where

$$
G\left(\xi_{0}\right)=\xi_{0}+\frac{A 2^{\prime}\left(\xi_{0}\right)}{\int_{\xi_{0}}^{\infty} A i(t) d t}, \quad \xi_{0}=-\frac{e^{-i \frac{\pi}{\epsilon} s_{n}} s_{n} \omega_{1}}{\left(\gamma\left|\bar{\lambda}_{1}\right|\right)^{\frac{2}{3}}}
$$

The right hand side of (6.1) is obtained from the solution of the wall layer problem (3.13). The real and imaginary parts of (6.1) gives two equations which can be solved to obtain correction terms to the wavenumber and wave-angle for a given frequency $\omega_{1}$. With $\omega_{1}$ set to zero in (6.1) we obtain the linear neutral results of Hall (1985) for stationary cross-flow vortices. The case $\omega_{1} \neq 0$ case therefore is a generalisation of the Hall (1985) results to 'almost' stationary vortices. The function $G\left(\xi_{0}\right)$ in $(6.1)$ was calculated numerically and is shown in Figure 1. For large $\left|\omega_{1}\right|$ it is easy to show that

$$
\begin{aligned}
& G\left(\xi_{0}\right) \sim-\frac{\xi_{0} e^{-i \frac{\pi}{4} s_{n}}\left(\gamma\left|\bar{\lambda}_{1}\right|\right)^{\frac{1}{3}}}{\left|\omega_{1}\right|^{\frac{3}{2}}} \text { as } s_{n} \omega_{1} \rightarrow \infty \\
& G\left(\xi_{0}\right) \sim-\frac{51 s_{n} \xi_{0}\left(\gamma\left|\bar{\lambda}_{1}\right|\right)^{\frac{1}{3}} e^{i s_{n} \frac{\pi}{4}}}{72 \imath\left|\omega_{1}\right|^{\frac{3}{2}}} \text { as } s_{n} \omega_{1} \rightarrow-\infty
\end{aligned}
$$

This implies that the influence of the wall layer on the cross-flow vortex diminishes as the scaled frequency increases.

(b) If we consider a disturbance of the form $A=\hat{A}(T) e^{\imath\left(\alpha_{1} X+\beta_{1} Z-\imath \omega_{1} T\right)}$ with $\beta_{1} \cdot \omega_{1}$ real and $\alpha_{1}$ complex, substitution into (6 1 ) shows that

$$
-\imath \alpha_{1}=\left[-\imath \frac{\omega \bar{I}_{1}}{\gamma}+\imath \frac{\beta_{1} \bar{I}_{2}}{\gamma}+\imath \frac{e^{\imath \frac{\pi}{\varepsilon} s_{n}} G\left(\xi_{0}\right)}{\left(\vartheta\left|\bar{\lambda}_{1}\right|\right)^{\frac{1}{3}}}\right] \frac{1}{\gamma_{i} I_{3}}
$$

Since $\bar{I}_{1}, \bar{I}_{2}$ are complex it can be seen that disturbances of this type are unstable with the growth rate increasing indefinitely for-large $\left|\beta_{1}\right|$ or $\left|w_{1}\right|$

(c) Consider a disturbance of the form $A=\tilde{A}(Z) e^{2\left(\alpha_{1} X-\omega_{1} T\right)}$, where $\alpha_{1}, \omega_{1}$ are real. This form of disturbance could, for instance, represent a combination of waves of the type considered in section (a) above. In addition this is a special case relevant to rotating disk 
flow, (for example if we take $B=\hat{B}(R) \epsilon^{2\left(\beta_{1} \Theta-\omega_{1} T\right)}$, with $\gamma_{1}$. wi leal. in $(A 6)$ of Appendix A) The equation (6 1$)$ then reduces to

$$
\frac{d \dot{A}}{d Z}=\kappa \tilde{A}-\frac{\kappa}{\phi} \bar{J}
$$

where

$$
\begin{aligned}
& \bar{J}=\left[\int_{0}^{\infty} d s_{3} \int_{0}^{\infty} d s_{2} s_{3}^{3} K_{4}^{(0)}\left(s_{2}, s_{3} \mid \tilde{\sigma}\right) \tilde{A}\left(Z-s_{3}\right) \tilde{A}\left(Z-s_{2}-s_{3}\right) \frac{d \tilde{A}^{(c)}}{d Z}\left(Z-s_{2}-2 s_{3}\right)\right. \\
& +\int_{0}^{\infty} d s_{3} \int_{0}^{\infty} d s_{2} s_{3}^{2} s_{2} H_{4}^{-(0)}\left(s_{2}, s_{3} \mid \tilde{\sigma}\right) \tilde{A}\left(Z-s_{3}\right) \frac{d}{d Z}\left[\tilde{A}\left(Z-s_{3}-s_{2}\right) \tilde{A}^{(c)}\left(Z-s_{2}-2 s_{3}\right)\right] \\
& +\int_{0}^{\infty} d s_{3} \int_{0}^{\infty} d s_{2} s_{3}^{3} K_{4}^{(0)}\left(s_{2}, s_{3} \mid \tilde{\sigma}\right) \frac{d}{d Z}\left[\tilde{A}\left(Z-s_{3}\right) \tilde{A}\left(Z-s_{2}-s_{3}\right) \tilde{A}^{(c)}\left(Z-s_{2}-2 s_{3}\right)\right],
\end{aligned}
$$

and we have set

$$
\kappa=\frac{\gamma}{\bar{I}_{2}}\left[\frac{\imath \omega_{1} \bar{I}_{1}}{\gamma}-\imath \alpha_{1} \gamma I_{3}-\imath \frac{e^{2 \frac{\pi}{\epsilon} s_{n}} G\left(\xi_{0}\right)}{\left(\gamma\left|\bar{\lambda}_{1}\right|\right)^{\frac{1}{3}}}\right], \quad \frac{\phi}{\kappa}=-\frac{\bar{I}_{2}\left|\mu_{0}\right|^{5}}{M}, \tilde{\sigma}=\frac{\sigma}{\left|\mu_{0}\right|^{3}} .
$$

As in Goldstein \& Leib (1989) it is found that the numerical solution of (6.3) points to a singularity as $Z \rightarrow Z_{s}$ A local asymptotic description of the singularity can be found by writing

$$
\tilde{A}=\frac{a}{\left(Z_{s}-Z\right)^{\tau}}
$$

with $a, \tau$ complex constants When $(66)$ is substituted into (6.3) it is seen that for a balance of the dominant terms on the left and right hand sides of the equation we require $\tau=\frac{5}{2}+2 \tau_{0}$, where $\tau_{0}$ is real. This leads to

$$
\bar{J} \sim \frac{a|a|^{2}}{\left(Z_{s}-Z\right)^{\tau+1}} D\left(\tau_{0}\right)
$$

where the function $D\left(\tau_{0}\right)$ is given in Appendix $\mathrm{B}$ and $D\left(\tau_{0}\right)$ satisfies the equation

$$
\frac{D\left(\tau_{0}\right)}{\tau}=-\frac{\phi}{|a|^{2} \kappa}
$$

In Figures 2,3 we show $\tau_{0}$ and $|a|$ as computed using (6 S) and (B1) as a function of $-\arg \left(\frac{\phi}{\kappa}\right)=\arg \left(\frac{D\left(\tau_{0}\right)}{\tau}\right)$ In Figures 2,3 we have taken $|\phi|=|\kappa|=1$ since as in Goldstein $\&$ Leib (1989) equation (6 3) is completely characterised by the arguments of $\phi$ and $\kappa$

\subsection{Numerical Solution of equation (6.3)}

The equation (6 3) was solved with the initial condition

$$
\tilde{A} \rightarrow \epsilon^{\wedge} \quad \text { as } Z \rightarrow-\infty .
$$

Here we have assumed that $\mu_{0}$ is positive The form of the arguments in the kernal function would suggest an apparent difficulty when $\mu_{0}$ is negatrve It is seen that when $\mu_{0}$ is negative the nonlinear terms in equation (6 3), (64) imply that the amplitude at 
the current $Z$ location is calculated from a knowledge of $\dot{A}$ at positions ahead of $Z$, in contrast to the case when $\mu_{0}$ is position when the calculation of $A(Z)$ involves history dependent effects This apparent difficulty can howeve be resolved by redefining $\theta$ used in (2 2), (by for example adding a multıple of $\pm \pi$ as thıs does not affect the subsequent criteria used to fix $\theta$ ) Similar comments apply also to the rotating disk flow and the appropriate choice for $\mu_{0}$ there, see (A6), (A7), is to take $\mu_{0}$ to be negative.

Equation (6.3) was solved numerically using a 5th ordel Adams Bashforth predictorcorrector scheme. The integrals were truncated to those over a finite domain and evaluated using a trapezoidal rule One novel feature of the present implementation of the method worth mentioning is that the computations were performed on a massively parallel computer, the Maspar MP-1104 which has 4096 processois arranged in a (64x64) square matrix. The bulk of the computational time in solving (6.3) numerically arises from the evaluation of the integrals especially when the nonlnnear terms become significant. Integrals of the type occurring in (6.3) can be evaluated extremely efficiently in parallel. Further detalls of the algorithm used may be obtained from the author.

Some solutions of (6.3) obtained numerically are shown in Figures 4-6. In Figure $4(\mathrm{a}-\mathrm{c})$ results are presented for the case with $\arg (\kappa)=0$ and $\arg (\dot{\phi})=-\pi / 2$ for values of $\tilde{\sigma}=0,0.5$, and 5 In these figures the dashed line is obtained from the asymptotic solution (6.6). The computations show that the location of the singularity is delayed with increasing $\tilde{\sigma}$. As the singular point is approached there is a sharp reduction in the wavenumber, Figure 4(b), accompanied by a very laige increase in the growth rate, Figure 4(c). The main solution characteristics for this set of parameters is broadly in line with those found by other investigators in their studies of related integro-differential equations.

The solutions presented in Figures 5(a-f), with $\arg (\kappa)=0, \arg (\phi)=-\pi / 4$ and $\tilde{\sigma}=$ $0,0.5$ and 5 , however, show a number of new and interesting properties, some of which have not been found before. The results for $\tilde{\sigma}=0$ and 0.5 are similar to those in Figure $4(\mathrm{a}-\mathrm{c})$ and show again that the singularity is delayed with increasing $\tilde{\sigma}$. For $\tilde{\sigma}=5$ on the other hand, our results, up to the largest $Z$ value that we have been able to compute, indicate that the singularity has been eliminated in favour of a large amplitude nonlinear oscillation. Figure 5d shows that the wavenumber fluctuates and contains a large high frequency component causing the wavenumber to peak at specific locations. The growth rate is seen in Figures 5(a), 5(e), to oscillate about zero, with again very large peaks near specific locations. In Figure 5(f) we show $|A|$ against $Z$ and this shows clearly the development of the nonlinear oscillations after an initially exponentially growing linear phase. This type of solution has not been found in studies of other related integrodifferential equations.

In Figure 6(a-c) we present results for the case $\arg (\kappa)=0$, and $\arg (\phi)=\pi / 4$ for $\tilde{\sigma}=$ 1 and 5. The solution properties are broadly similar to those for the case with $\phi=-\pi / 4$, except that the wavenumber has large negative peaks at certain locations. The real part of the growth rate for $\tilde{\sigma}=5$ is exactly the same as that in Figure $5(\mathrm{e})$.

The comparisons between the asymptotic and the numerical results in Figures 4-6 are quite good and this indicates that the correct singularity structure has been captured. In plotting the asymptotic predictions, for a given value of $\arg \left(D\left(\tau_{0}\right) / \tau\right)$ the value of $\tau_{0}$ and $a$ were obtained fiom (63), with the value of $Z_{s}$ extrapolated from the numerical results

The numerical solutions take an extremely long time to compute. expecially when the 
nonlinear terms become significant Fuither more extensive calculations are currently in progress to explore a wider range of parameter values. and to see whether the nonlinear waveform foi $\mathrm{m}$ found for some parameter values, persists ol is damped out for large $Z$

\section{Further discussion and conclusions}

In this paper we have obtained a novel integro-differential equation which describes the nonlinear evolution of stationary cross-flow vortices in thiee-dimensional incompressible boundary layer flows. It has been shown that the evolution of the vortex depends crucially on the dynamics of the unsteady critical layer as well as the dynamics of an unsteady wall layer. In companion papers the work presented here is extended to non-stationary cross-flow vortıces, Gajjar \& Arebı (1985), and to complessıble flows Gajjar \& Sibanda (1985). In Gajjar \& Arebi (1985) it is found that the amplitude of the non-stationary vortex satisfies a simular equation but without the wall coupling present. In addition this equation has an additional Hickernell (1984) type term whose coefficient depends on the curvature of the effective velocity profile at the critical layer. The influence of the wall layer in the current problem diminishes as the the magnitude of the scaled frequency increases as was shown in the previous section.

The amplitude equation has a number of interesting properties some of which have been discussed already. The full problem (5.4) is of considerable interest and merits further study both analytically and numerically. As in many related problems it has been shown that solutions to the amplitude equation can develop finite-distance singularities. A preliminary analysis of (5.4) suggests that focussing type singularities of the form

$$
A(X, Z, T) \sim e^{-\imath \omega_{1} T} \frac{F(\hat{X})}{\left(Z_{s}-Z\right)^{\frac{5}{2}+\imath \tau_{0}}}, \quad \hat{X}=\frac{\left(X-X_{s}\right)}{\left(Z_{s}-Z\right)}
$$

may also exist. The function $F(\hat{X})$ satisfies a nonlinear first order integro-differential equation which can be written down, see also $\mathrm{Wu}(1994)$

The coupling with the wall layer found here is impoitant in another context, namely the study of the receptivity of stationary cross-flow vortices to suiface mounted obstacles. Experimentally it has been observed that even minute roughness elements can act as a trigger for stationary vortices, see Wilkinson et al (19S3), Reed \& Saric (1989). It is suggested that the close coupling with the wall layer and the manner in which this affects the evolution of a cross-flow vortex, may in fact provide a simple explanation for this phenomenon. The scales and structure presented here may be used to study this aspect in more detall.

Solutions of the amplitude equation in which the finite-distance, or focussing type, singularities form, although mathematically interesting do not however tie in with the observations in some experiments and numerical simulations of a nonlinear saturation of stationary and non-stationary cross-flow vortices In this repect some of the other solutions shown in the previous section, in which a nonlinear wave develops, may have more relevance. Other possible equilibrium solutions of the equation are currently being investigated. With other scalings, see Gajjar (1994), it has been shown that the evolution of long wavelength cross-flow vortices is governed by the full unsteady nonlinear critical layers equations In many related problems where similar equations arise. it is typically found that the growth rate of the disturbances is driven to zero Thus this type of 
critical layer nonlinearity may also pioride an explanation for the nonlinear saturation of the vortices

Stuart in GSW (1955) found that the number of roltices predicted by the linear inviscid theory was much greater than that observed in thell experiments. Although a number of suggestions have been made to account for this disciepancy; our computations show that nonlinearity provides a wavelength increasing/decieasing mechanism. On the other hand the flow in the neighbourhood of the singularity, where the wavenumber is changed by an $O(1)$ amount, is no longer governed by linear dynamics, but rather the full Euler equations. A detalled comparison with experimental and other data is clearly desirable but requires substantial further work A simple evaluation of some of the constants arising from the linear inviscid eigenvalue problem (3.4) is clearly not sufficient.

\section{Acknowledgement}

The author is grateful to Dr's M.E. Goldstein, L. Hultgren, S. Leib, and D. Wundrow, for many helpful discussions. Dr S.J. Cowley is thanked for pointing out an error in an earlier formulation of the problem. The author gratefully acknowledges the support of ICOMP, NASA Lewis Research Center, Cleveland, where part of this work was done during a visit in summer of 1993 , and to E.P.S.R.C. for providing computing equipment used in this work through grants GR/H58565-C88 and GR/H 83683. 


\section{Appendix A}

In this appendix we consides the flow over a rotating dish and derw e the corresponding amplitude equation for a stationary cross-flow vortex

Consider a disk which rotates about the $z$ axis with angulai velocity $\Omega$. Relative to cylindrical polar corrdinates $(r, \theta . z)$ which rotate with the disk, the continuity and Navier-Stokes equations, suitably non-dimensionalised. are

$$
\begin{gathered}
\nabla \mathbf{u}=0 \\
\frac{\partial \mathbf{u}}{\partial t}+(\mathbf{u} \nabla) \mathbf{u}+2(\mathbf{k} \times \mathbf{u})-r \hat{\mathbf{r}}=-\nabla p+\frac{1}{R} \nabla^{2} \mathbf{u},
\end{gathered}
$$

where $\mathbf{u}=(u, w, v)$ are the velocity components, $p$ is the pressure, $\hat{\mathbf{r}}$ and $\mathbf{k}$ are unit vectors in the $r-$ and $z$-coordinate directions, and $R$ is the Reynolds number. The Reynolds number is taken to be large. With $\epsilon=R^{-\frac{1}{\varepsilon}}$ and $z=\epsilon^{3} Y$. the basic flow is given by Von-Karman's exact solution of the Narier-Stokes equation, $(u, w, v)=$ $\left(\bar{u}(Y), r \bar{w}(Y), \epsilon^{3} \bar{v}(Y)\right), p=\bar{p}(Y)$ where $\bar{u}, \bar{w}, \bar{v}, \bar{p}$ satisfy

$$
\begin{gathered}
\bar{u}^{2}-(1+\bar{w})^{2}+\bar{u}^{\prime} \bar{v}=\bar{u}^{\prime \prime}, \quad 2 \bar{u}(1+\bar{w})+\bar{w}^{\prime} \bar{v}=\bar{w}^{\prime \prime}, \\
\bar{v}^{\prime}+2 \bar{u}=0, \quad \bar{p}^{\prime}+\bar{v} \bar{v}^{\prime}-\bar{v}^{\prime \prime}=0
\end{gathered}
$$

The boundary conditions for $(\bar{u}, \bar{w}, \bar{v})$ are

$$
\begin{gathered}
\bar{u}=\bar{w}=\bar{v}=0 \quad \text { on } \quad Y=0, \\
\bar{u}=0, \quad \bar{w} \rightarrow-1 \quad \text { as } \quad Y \rightarrow \infty
\end{gathered}
$$

Next consider a stationary cross-flow vortex at a location $\left(r_{0}, \theta\right)$ and introduce a multiple-scaling as in section 2 such that

$$
\begin{gathered}
\frac{\partial}{\partial r} \rightarrow \epsilon^{-3}\left[\alpha_{0} \frac{\partial}{\partial \xi}+\epsilon \frac{\partial}{\partial R}\right]+\frac{\partial}{\partial r}, \\
\frac{\partial}{\partial \theta} \rightarrow \epsilon^{-3}\left[\beta_{0} \frac{\partial}{\partial \xi}+\epsilon \frac{\partial}{\partial \Theta}\right], \quad \frac{\partial}{\partial t} \rightarrow \epsilon^{-2} \frac{\partial}{\partial T} .
\end{gathered}
$$

Here $\alpha_{0}=\cos \bar{\theta}_{0}, \quad \beta_{0} / r_{0}=\sin \bar{\theta}_{0}$, and $\bar{\theta}_{0}$ is chosen such that $\left(\alpha_{0} \bar{u}+\left(\beta_{0} / r_{0}\right) \bar{w}\right)$ has a zero at a point of inflexion This also fixes the location of the cutical level $Y^{\prime}=Y_{c}$ The expansions for the flow quantities are similar to (3.1) with

$$
\begin{aligned}
u & =\bar{u}+\delta\left(\overline{\bar{u}}_{0}+\epsilon \overline{\bar{u}}_{1}+.\right), \\
w & =r \bar{w}+\delta\left(\overline{\bar{w}}_{0}+\epsilon \overline{\bar{w}}_{1}+.\right), \\
v & =\epsilon^{3} \bar{v}+\delta\left(\overline{\bar{v}}_{0}+\epsilon \overline{\bar{v}}_{1}+.\right), \\
p & =\bar{p}+\delta\left(\overline{\bar{p}}_{0}+\epsilon \overline{\bar{p}}_{1}+.\right) .
\end{aligned}
$$

After substituting (A3) into (A1) we obtain

$$
\begin{gathered}
\alpha_{0} \overline{\bar{u}}_{0 \xi}+\frac{\beta_{0}}{r_{0}} \overline{\bar{w}}_{0 \xi}+\overline{\bar{v}}_{0 Y}=0, \\
\alpha_{0} \overline{\bar{u}}_{1 \xi}+\overline{\bar{u}}_{0 R}+\frac{\beta_{0}}{r_{0}} \overline{\bar{w}}_{1 \xi}+\frac{1}{r_{0}} \overline{\bar{w}}_{0 \Theta}+\overline{\bar{v}}_{1 Y}=0
\end{gathered}
$$




$$
\begin{aligned}
& \left(a_{0} \bar{u}+\frac{\beta_{0}}{r_{0}} \bar{u}\right) \overline{\bar{u}}_{0 \xi}+\overline{\bar{v}}_{0} \bar{u}_{Y}=-a_{0} \overline{\bar{p}}_{0 \xi} . \\
& \left(a_{0} \bar{u}+\frac{\beta_{0}}{r_{0}} \bar{u}\right) \overline{\bar{u}}_{0 \xi}+\overline{\bar{v}}_{0} \bar{w}_{Y^{\prime}}=-\frac{3_{0}}{r_{0}} \overline{\bar{p}}_{0 \xi} . \\
& \left(a_{0} \bar{u}+\frac{3_{0}}{r_{0}} \bar{w}\right) \overline{\bar{v}}_{0 \xi}=-\overline{\bar{p}}_{0 \nu}, \\
& \overline{\bar{u}}_{0 T}+\left(\alpha_{0} \bar{u}+\frac{\beta_{0}}{r_{0}} \bar{w}\right) \overline{\bar{u}}_{1 \xi}+\bar{u} \overline{\bar{u}}_{0 R}+\frac{\bar{w}}{r_{0}} \overline{\bar{u}}_{0 \Theta}+\overline{\bar{v}}_{1} \bar{u}_{Y^{\prime}}=-a_{0} \overline{\bar{p}}_{1 \xi}-\overline{\bar{p}}_{0 R}, \\
& \overline{\bar{w}}_{0 T}+\left(\alpha_{0} \bar{u}+\frac{\beta_{0}}{r_{0}} \bar{w}\right) \overline{\bar{w}}_{1 \xi}+\bar{u} \overline{\bar{w}}_{0 R}+\frac{\bar{w}}{r_{0}} \overline{\bar{w}}_{0 \Theta}+\overline{\bar{v}}_{1} \overline{\bar{w}}_{I}=-\frac{\beta_{0}}{r_{0}} \overline{\bar{p}}_{1 \xi}-\frac{1}{r_{0}} \overline{\bar{p}}_{0 \Theta},
\end{aligned}
$$

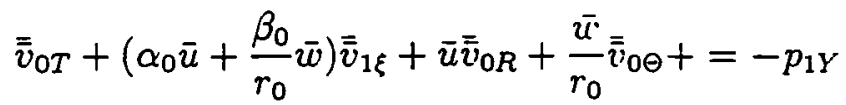

Next if we define

$$
\begin{gathered}
X=\alpha_{0} R+\beta_{0} \Theta, \quad Z=\frac{-\beta_{0}}{r_{0}} R+\alpha_{0} r_{0} \Theta, \\
U_{B}=\alpha_{0} \bar{u}+\frac{\beta_{0}}{r_{0}} \bar{w}, \quad W_{B}=-\frac{\beta_{0}}{r_{0}} \bar{u}+\alpha_{0} \bar{w} . \\
\bar{u}_{k}=a_{0} \overline{\bar{u}}_{k}+\frac{\beta_{0}}{r_{0}} \overline{\bar{w}}_{k}, \quad \bar{w}_{k}=-\frac{\beta_{0}}{r_{0}} \overline{\bar{u}}_{h}+\alpha_{0} \overline{\bar{u}}_{h},
\end{gathered}
$$

then using (A4), (A5) it is found that the equations for $\left(\bar{u}_{0} . \bar{v}_{0} . \bar{v}_{0}\right),\left(\bar{u}_{1}, \bar{v}_{1}, \bar{w}_{1}\right)$ are identical to (3.2) and (3.7). We can thus introduce a similar normal mode decomposition so that for example,

$$
\bar{u}_{0}=A(X, T, Z) \tilde{U}_{0}(Y) e^{\imath\urcorner} \xi+\mathrm{c} c
$$

The expansions for the wall layer and critical layer follow analogously. Thus in the critical layer where $z=\epsilon^{3} Y_{c}+\epsilon^{4} \eta$ we have

$$
\begin{aligned}
u & =\bar{u}+\epsilon^{\frac{3}{2}} \hat{u}_{0}+\epsilon^{2} \hat{u}_{1}+\epsilon^{\frac{5}{2}} \hat{u}_{2}+\epsilon^{3} \hat{u}_{3}+\epsilon^{\frac{7}{2}} \hat{u}_{4}+\ldots, \\
w & =r \bar{w}+\epsilon^{\frac{3}{2}} \hat{w}_{0}+\epsilon^{2} \hat{w}_{1}+\epsilon^{\frac{5}{2}} \hat{w}_{2}+\epsilon^{3} \hat{u}_{3}+\epsilon^{\frac{7}{2}} \hat{u}_{4}+. \\
v & =\epsilon^{3} \bar{v}+\epsilon^{\frac{5}{2}} \hat{v}_{-1}+\epsilon^{\frac{7}{2}} \hat{v}_{0}+\epsilon^{4} \hat{v}_{1}+\epsilon^{\frac{9}{2}} \hat{v}_{2}+\ldots \\
p & =\bar{p}+\epsilon^{\frac{5}{2}} \hat{p}_{0}+\epsilon^{3} \hat{p}_{1}+\epsilon^{\frac{7}{2}} \hat{p}_{2}+\epsilon^{4} \hat{p}_{3}+\epsilon^{\frac{3}{2}} \hat{p}_{4}+\ldots
\end{aligned}
$$

If we define

$$
\lambda_{k}=\left[\alpha_{0} \frac{d^{k}}{d r^{k}}(\bar{u})+\frac{\beta_{0}}{r_{0}} \frac{d^{k}}{d r^{k}} \bar{w}\right]_{Y^{\prime}=Y_{c}}, \quad \mu_{k}=\left[-\frac{\beta_{0}}{1_{0}} \frac{d^{k}}{d r^{k}} \bar{u}+a_{0} \frac{d^{k}}{d r^{k}} \bar{w}\right]_{Y^{\prime}=Y_{c}},
$$

and

$$
\bar{U}_{k}=\alpha_{0} \hat{u}_{k}+\frac{\beta_{0}}{r_{0}} \hat{w}_{k}, \quad \bar{W}_{k}=-\frac{\beta_{0}}{r_{0}} \hat{u}_{k}+\alpha_{0} \hat{w}_{k} . \quad \bar{V}_{k}=\hat{\imath}_{k} . \quad \bar{P}_{k}=\hat{p}_{k}
$$

then to the required or $\operatorname{der}\left(\bar{U}_{k}, \bar{V}_{k}, \bar{W}_{k}, \bar{P}_{k}\right)$ satisfy the same equations as the corresponding variables in section 4 The amplitude equation, in terms of $A$. is therefore identical to (5.4). In terms of $R, \Theta$ variables however, if we define the scaled amplitude of the crossflow vortex as $B(R, T, \Theta)$ where

$$
B(R, T, \Theta)=A(\mathcal{X}, T . Z)
$$


and use the definitions of $\mathcal{I}^{-} Z$ from $(A j)$, then the amplitude equation is grven by

$$
\frac{\bar{I}_{1}}{\gamma} \frac{\partial B}{\partial T}+\frac{\bar{I}_{2}}{\gamma}\left(-\frac{\beta_{0}}{r_{0}} \frac{\partial B}{\partial R}+\frac{\alpha_{0}}{r_{0}} \frac{\partial B}{\partial \Theta}\right)+\gamma I_{3}\left(\alpha_{0} \frac{\partial B}{\partial R}+\frac{\beta_{0}}{r_{0}^{2}} \frac{\partial B}{\partial \Theta}\right)=\left[\check{I}_{0 \gamma} i_{i}\right]_{\}=0}+M\left(J_{1}+J_{2}+J_{3}\right) \text {. }
$$

where

$$
\begin{gathered}
J_{1}=\int_{0}^{\infty} d s_{3} \int_{0}^{\infty} d s_{2} s_{3}^{3} B\left(R+\frac{\beta_{0} \mu_{0} s_{3}}{r_{0}}, T-s_{3}, \Theta-\frac{a_{0} \mu_{0} s_{3}}{r_{0}}\right) K_{4}^{(0)}\left(s_{2}, s_{3} \mid \sigma\right) \times \\
B\left(R+\frac{\mu_{0} \beta_{0}}{r_{0}}\left(s_{2}+s_{3}\right), T-s_{2}-s_{3}, \Theta-\mu_{0} \frac{a_{0}\left(s_{2}+s_{3}\right)}{r_{0}}\right)\left(-\frac{\beta_{0}}{r_{0}} \frac{\partial}{\partial R}+\frac{a_{0}}{r_{0}} \frac{\partial}{\partial \Theta}\right) \times \\
\quad\left[B^{(c)}\left(R+\frac{\mu_{0} \beta_{0}}{r_{0}}\left(s_{2}+2 s_{3}\right) \cdot T-s_{2}-2 s_{3}, \Theta-\frac{\mu_{0} a_{0}}{r_{0}}\left(s_{2}+2 s_{3}\right)\right)\right] \\
J_{2}=\int_{0}^{\infty} d s_{3} \int_{0}^{\infty} d s_{2} s_{3}^{2} s_{2} B\left(R+\frac{\beta_{0} \mu_{0}}{r_{0}} s_{3}, T-s_{3}, \Theta-\frac{a_{0} \mu_{0}}{r_{0}} s_{3}\right) K_{4}^{(0)}\left(s_{2}, s_{3} \mid \sigma\right) \times \\
\left(-\frac{\beta_{0}}{r_{0}} \frac{\partial}{\partial R}+\frac{\alpha_{0}}{r_{0}} \frac{\partial}{\partial \Theta}\right)\left[B\left(R+\frac{\beta_{0} \mu_{0}}{r_{0}}\left(s_{2}+s_{3}\right), T-s_{3}-s_{2}, \Theta-\frac{\alpha_{0} \mu_{0}}{r_{0}}\left(s_{3}+s_{2}\right)\right)\right. \\
\left.B^{(c)}\left(R+\frac{\mu_{0} \beta_{0}}{r_{0}}\left(s_{2}+2 s_{3}\right), T-s_{2}-2 s_{3}, \Theta-\frac{\mu_{0} a_{0}}{r_{0}}\left(s_{2}+2 s_{3}\right)\right)\right] \\
J_{3}=\int_{0}^{\infty} d s_{2} \int_{0}^{\infty} d s_{1} s_{2}^{3} K_{4}^{(0)}\left(s_{1}, s_{2} \mid \sigma\right)\left(-\frac{\beta_{0}}{r_{0}} \frac{\partial}{\partial R}+\frac{\alpha_{0}}{r_{0}} \frac{\partial}{\partial \Theta}\right)\left[B\left(R+\frac{\mu_{0} \beta_{0}}{r_{0}} s_{2}, T-s_{2}, \Theta-\frac{\alpha_{0} \mu_{0}}{r_{0}} s_{2}\right) \times\right. \\
B\left(R+\frac{\mu_{0} \beta_{0}}{r_{0}}\left(s_{2}+s_{1}\right), T-s_{2}-s_{1}, \Theta-\frac{a_{0} \mu_{0}}{r_{0}}\left(s_{2}+s_{1}\right)\right) \times \\
\left.B^{(c)}\left(R+\frac{\mu_{0} \beta_{0}}{r_{0}}\left(2 s_{2}+s_{1}\right), T-2 s_{2}-s_{1}, \Theta-\frac{a_{0} \mu_{0}}{r_{0}}\left(2 s_{2}+s_{1}\right)\right)\right] .
\end{gathered}
$$




\section{Appendix B}

The function $D\left(\tau_{0}\right)$ is defined as follows with $\tau=\frac{i}{2}+i \tau_{0}$

$$
D\left(\tau_{0}\right)=-2 \tau^{(c)} K_{1}^{\prime}+\tau\left(K_{21}^{\prime}+K_{22}\right)-\tau^{(\Leftrightarrow)}\left(K_{23}^{\prime}+K_{24}\right)-\tau\left(K_{31}+K_{32}^{\prime}\right),
$$

where

$$
\begin{gathered}
K_{1}^{\prime}=-\int_{0}^{\infty} d p \int_{p}^{\infty} d q \frac{(p-1)^{3}}{p^{\tau} q^{\tau}(p+q-1)^{\tau^{(c)}+1}} \\
K_{21}=\int_{0}^{\infty} d p \int_{p}^{\infty} d q \frac{(p-1)^{2}}{p^{\tau} q^{\tau}(p+q-1)^{\tau^{(c)}}} \\
K_{22}=-\int_{0}^{\infty} d p \int_{p}^{\infty} d q \frac{(p-1)^{2}}{p^{\tau-1} q^{\tau+1}(p+q-1)^{\tau^{(c)}}} \\
K_{23}=\int_{0}^{\infty} d p \int_{p}^{\infty} d q \frac{(p-1)^{2}}{p^{\tau} q^{\tau-1}(p+q-1)^{\tau^{(c)}+1}} \\
K_{24}=-\int_{0}^{\infty} d p \int_{p}^{\infty} d q \frac{(p-1)^{2}}{p^{\tau-1} q^{\tau}(p+q-1)^{\tau^{(c)}+1}} \\
K_{31}=\int_{0}^{\infty} d p \int_{p}^{\infty} d q \frac{(p-1)^{3}}{p^{\tau+1} q^{\tau}(p+q-1)^{\tau^{(c)}}} \\
K_{32}=\int_{0}^{\infty} d p \int_{p}^{\infty} d q \frac{(p-1)^{3}}{p^{\tau} q^{\tau+1}(p+q-1)^{\tau^{(c)}}} .
\end{gathered}
$$

These integrals can be evaluated as in Goldstein \& Leib(1989) to give

$$
\begin{aligned}
& K_{1}=\sum_{n=1}^{\infty} \frac{(-1)^{(n+1)}}{n(n+1)} \frac{1}{\left(\frac{7}{2}+\imath \tau_{0}\right)_{n}}\left[\left(-\frac{1}{2}-\imath \tau_{0}\right)_{n}-4\left(\frac{1}{2}-\imath \tau_{0}\right)_{n}+6\left(\frac{3}{2}-2 \tau_{0}\right)_{n}\right. \\
& \left.-4\left(\frac{5}{2}-2 \tau_{0}\right)_{n}+\left(\frac{7}{2}-\imath \tau_{0}\right)_{n}\right] \\
& K_{21}^{\prime}=\Delta_{\frac{3}{2}}^{(2)}\left(\tau_{0}\right)-3 \Delta_{\frac{1}{2}}^{(2)}\left(\tau_{0}\right) \text {, } \\
& K_{22}^{\prime}=\sum_{n=2}^{\infty} \frac{(-1)^{(n+1)}}{n(n+1)(n-1)} \frac{\tau}{\left(\frac{5}{2}+\imath \tau_{0}\right)_{n}}\left[\left(-\frac{3}{2}-\imath \tau_{0}\right)_{n}-4\left(-\frac{1}{2}-\imath \tau_{0}\right)_{n}+6\left(\frac{1}{2}-\imath \tau_{0}\right)_{n}\right. \\
& \left.-4\left(\frac{3}{2}-\imath \tau_{0}\right)_{n}+\left(\frac{5}{2}-\imath \tau_{0}\right)_{n}\right] \\
& K_{23}=\sum_{n=1}^{\infty} \frac{(-1)^{n}}{n(n+1)} \frac{1}{\left(\frac{7}{2}+\imath \tau_{0}\right)_{n}}\left[\left(\frac{1}{2}-\imath \tau_{0}\right)_{n}-3\left(\frac{3}{2}-\imath \tau_{0}\right)_{n}+3\left(\frac{5}{2}-\imath \tau_{0}\right)_{n}-\left(\frac{7}{2}-\imath \tau_{0}\right)_{n}\right] \text {, } \\
& K_{24}=\sum_{n=2}^{\infty} \frac{(-1)^{(n+1)}}{n(n-1)(n+1)} \frac{\tau}{\left(\frac{5}{2}+\imath \tau_{0}\right)_{n}}\left[\left(-\frac{1}{2}-\imath \tau_{0}\right)_{n}-4\left(\frac{1}{2}-\imath \tau_{0}\right)_{n}+6\left(\frac{3}{2}-\imath \tau_{0}\right)_{n}\right. \\
& \left.-4\left(\frac{5}{2}-2 \tau_{0}\right)_{n}+\left(\frac{7}{2}-\imath \tau_{0}\right)_{n}\right] \\
& K_{31}^{\prime}=\Delta_{\frac{3}{2}}^{(2)}\left(\tau_{0}\right)-3 \Delta_{\frac{1}{2}}^{(2)}\left(\tau_{0}\right) \text {, }
\end{aligned}
$$




$$
\begin{aligned}
K_{32}=\sum_{n=1}^{\infty} \frac{(-1)^{n}}{n(n+1)} \frac{1}{\left(\frac{7}{2}+\imath \tau_{0}\right)_{n}}[ & \left(-\frac{3}{2}-\imath \tau_{0}\right)_{n}-4\left(-\frac{1}{2}-\imath \tau_{0}\right)_{n}+6\left(\frac{1}{2}-2 \tau_{0}\right)_{n} \\
& \left.-4\left(\frac{3}{2}-2 \tau_{0}\right)_{n}+\left(\frac{j}{2}-\imath \tau_{0}\right)_{n}\right],
\end{aligned}
$$

where

$$
\begin{gathered}
\Delta_{r}^{(1)}\left(\tau_{0}\right)=\sum_{n=1}^{\infty} \frac{(-1)^{n}}{n(n+1)} \frac{1}{\left(\frac{7}{2}+\imath \tau_{0}\right)_{n}}\left[\left(1-r-\imath \tau_{0}\right)_{n}-\left(1+r-\imath \tau_{0}\right)_{n}\right], \\
\Delta_{r}^{(2)}\left(\tau_{0}\right)=\sum_{n=1}^{\infty} \frac{(-1)^{n}}{n(n+1)} \frac{1}{\left(\frac{7}{2}+\imath \tau_{0}\right)\left(\frac{9}{2}+\imath \tau_{0}\right)_{n}}\left[\left(1-r-\imath \tau_{0}\right)_{n}-\left(1+r-\imath \tau_{0}\right)_{n}\right],
\end{gathered}
$$

and $(a)_{n}$ denotes the function $\Gamma(a+n) / \Gamma(a)$. 


\section{References}

Arnal, D., (1986), Three-dimensional boundary layers-laminar-turbulent transition.

AGARD Rep. no $T 41$

Balakumar, P., \& Malık, M R, (1990), Travellıng disturbances in rotatıng disk flow. Theor. Comp. Fluid Dyn., 2, 125

Bassom, A P., \& Gajjar, J.S.B , (1988), Non-stationary cross-flow vortıces in a threedimensional boundary layer Proc. Roy. Soc., A 417, 179

Bippes, H., \& Nitchke-Kowsky, P., (1987) Experimental study of instabrlıty modes in a three-dimensional boundary layer. A.I.A A. pap no 87-1336

Cowley, S.J., \& Wu, X , (1994), Asymptotic approaches to transition modelling. AGARD Rep. no 793, Chap. 3, 1-38.

Gajjar, J.S.B., (1994), Nonlınear stabzlity of non-statzonary cross-flow vortzces in compressible boundary layers, submitted to Stud. Appl. Math.

Gajjar, J.S.B., (1995) (in preparation).

Gajjar, J.S.B., \& Arebi, M., (1995). Nonlinear spatial/temporal development of nonstationary cross-flow vortıces, (in preparation).

Gajjar, J.S.B., \& Sibanda, P., (1995), The nonlinear stabılıty of cross-flow vortices in compressible flows (in preparation).

Goldstein, M.E., (1994), Nonlinear interactions between oblique instabılıty waves on nearly parallel shear flows. Physics of Fluids, 6, (2), 724.

Goldstein, M.E., \& Chol, S.W., (1989), Nonlinear evolution of interactıng oblique waves on two-dimensional shear layers, J. Fluid Mech., 207, 97. Corrigendum, ibıd 216, 659.

Goldstein, M.E., \& Leib, S.J., (1989), Nonlinear evolution of oblique waves on compressible shear layers, J. Fluid Mech., 207, 73.

Gregory, N., Stuart, J.T., \& Walker, W.S., (1955), On the instability of three-dimensional boundary layers with application to the flow due to a rotating disk Phil Trans. Roy. Soc., A 248, 155.

Hall, P., (1986), An asymptotic investrgation of the stationary modes of instability of the boundary layer on a rotating disk. Proc. Roy. Soc. Lond., A 406, 93

Hickernell, F.J., (1984), Time-dependent critical layers in shear flows on the beta-plane. J. Fluid Mech., 142, 431.

Kobayashı, R., \& Kohama, Y., and Takamadate, Ch., (1980), Spıral vortıces in boundary layer transition regime on a rotating disk, Acta Mech., 35, 71

Kohama,Y. (1984), Study on boundary layer transition of a rotating disk, Acta Mech., $50,193$.

Kohama, Y., Saric, W.S., \& Hoos, J.A., (1991), A high-frequency secondary instabılıty of cross-flow vortices that leads to transition. in Proc Royal Aero Soc conf. on Boundary Layer Transition and Control, Cambridge. 4.1.

Leib, S.J, (1991), Nonlinear evolution of subsonıc and supersonic disturbances on a compressible mixıng layer J. Fluid Mech, 224, 551

Mackerrel, S O., (1987), A nonlinear asymptotic investigation of the stationary modes of instabilıty of the 3-dimensional boundary layer on a rotatıng disk, Proc Roy. Soc. A 413, 497.

Malik, M , (1986), The neutral curve for stationary disturbances in rotating disk flow. J Flusd Mech., 164, 275.

Malık, M., \& Li, F , (1992), Three-dimensional boundary layer stabılity and transition S.A.E Tech Pap no 921991. 
Malık, M \& \& L1, F , \& Chang. C.L., (1994), Crossflow disturbances in three-dimensional boundary layers nonlinear development, wave interaction and secondary instabllity, J Fluid Mech., 268, 1

Malık, M R., \& Wilkinson, S.P., and Oiszag. S.A., (1981), Instabilty and Transition in rotating disk flow, AIAA Jour.. 19, 1131

Meyer, F., \& Kileiser, L., (19S8), Numerical investigation of transition in $3 D$ boundary layers. Proc. AGARD Sysmposium on Fluid Dynamics of Three-Dimensional Turbulent Shear Flows and Transition, Cesme, Turkey

Michel, J.M., Arnal, D., Juillen, J.C., (1985), Experimantal and theoretical studies of boundary layer transition on a swept infinite wing, in Laminar and Turbulent Transition, (ed. V.V. Kozlov), Norosibirsk, Springer, 553.

Muller, B., \& Bippes, H., (1988), Experimental study of instabrlity modes in a threedimensional boundary layer. Proc. AGARD Sysmposium on Fluid Dynamics of ThreeDimensional Turbulent Shear FLows and Transition, Cesme, Turkey.

Poll, D.I.A., (1985), Some observations of the transition process on the windward face of a long yawed cylinder. J. Fluid Mech., 150, 329.

Reed, H.L., \& Sarıc, W.S., (1989), Stabzlity of three-dimensional boundary layers. Ann. Rev. Fluid Mech., 21235

Saric,W.S., \& Dagenhart, J.R., and Mousseux, M.C., (1989), Experiments in swept-wing transition, in Numerical and physical aspects of aerodynamic flows, 4 (ed. T. Cebec1), Springer-Verlag.

Wilkınson, S.P., \& Malik, M.R., (1983). Stabrlity experıments in rotatıng disk flow. AIAA Pap. no. 83-1760

Wu, X., (1992), Ph.D. Thesis, Unıv of London.

Wu, X., (1993), Nonlinear temporal-spatzal modulation of near-planar Rayleagh waves in shear flows: formation of streamwise vortıces J. Fluid Mech.. 256., 685. 

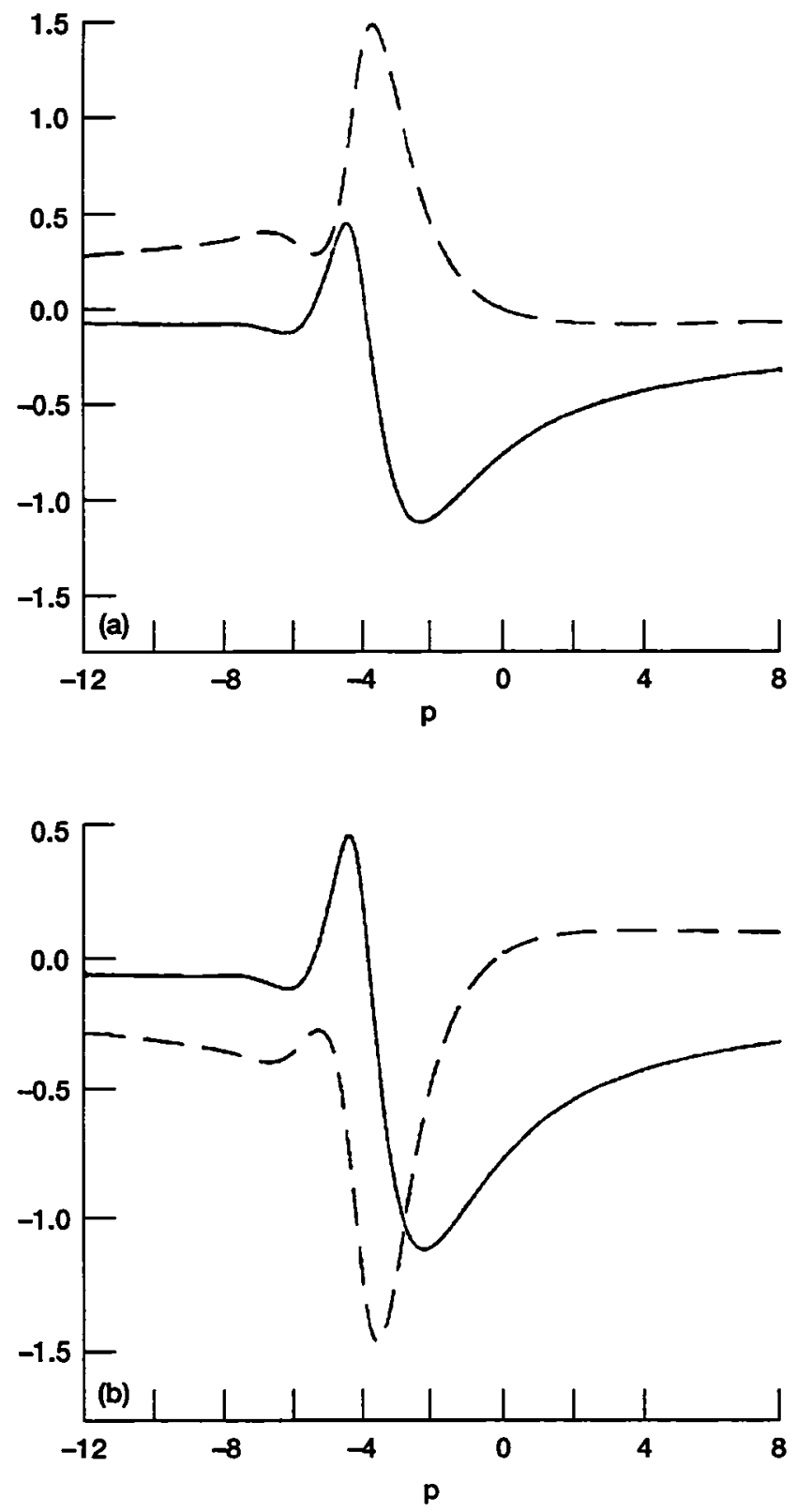

Figure 1. - (a) Real (soild IIne) and Imaginary (dashed Ine) parts of $G\left(e^{-1} \pi / 6 p\right)$ against p. (b) Real (solid line) and Imaginary (dashed line) parts of $G\left(e^{-1} \pi / 6 p\right)$ against $p$. 


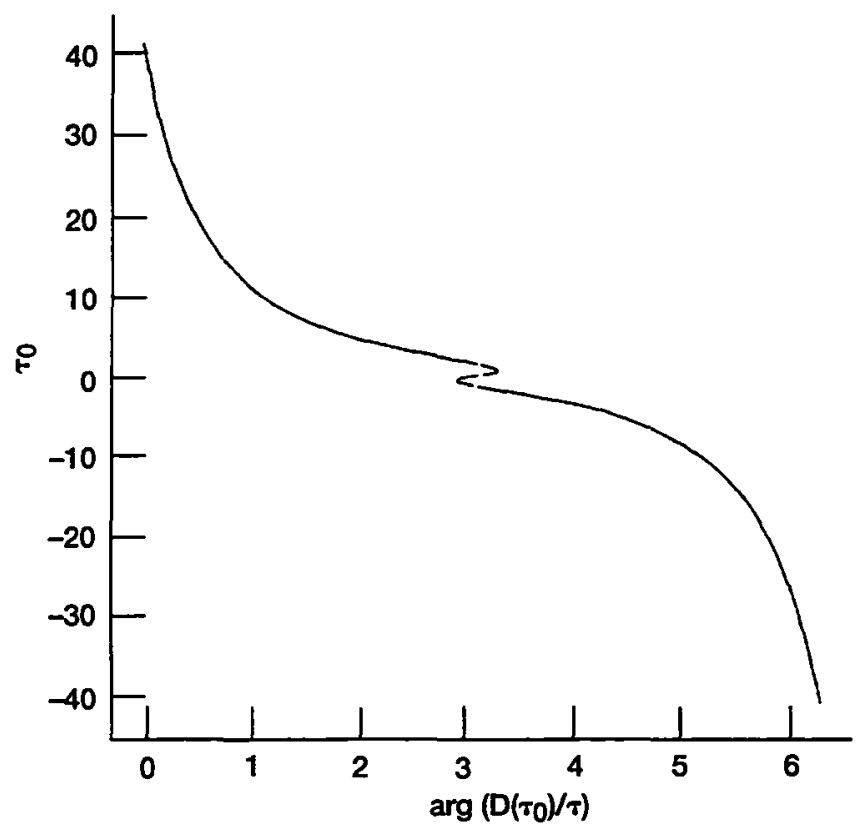

Figure 2.-Curve showing vanation of $\tau_{0}$ against $\arg \left(\mathrm{D}\left(\tau_{0}\right) / \tau\right)$.

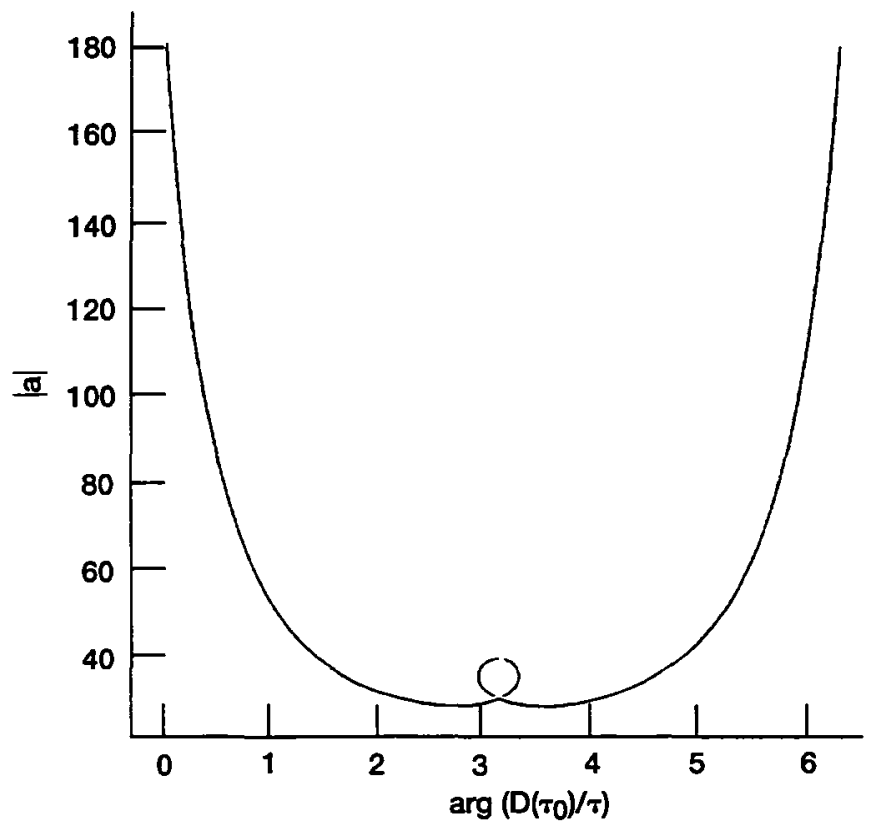

Figure 3.-Curve showing variation of $|\mathrm{a}|$ against $\arg \left(\mathrm{D}\left(\tau_{0}\right) / \tau\right)$ 

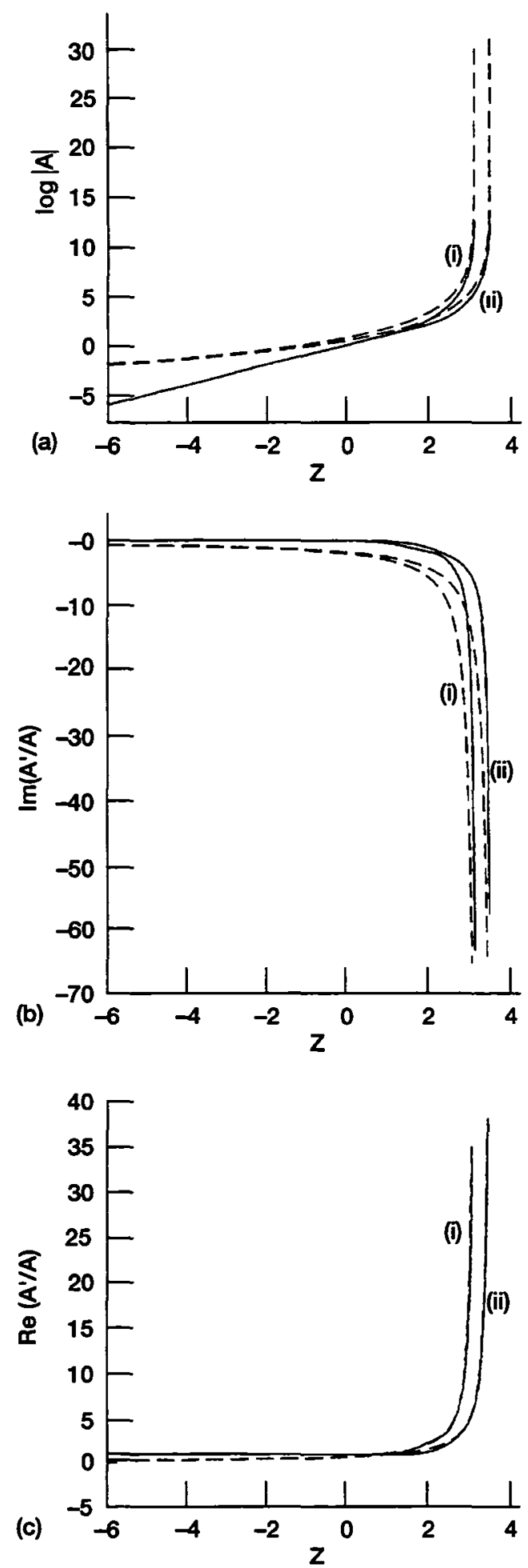

Fıgure 4.- (a) A plot of $\log \mid \tilde{\mathbf{A}}$ against $Z$ as calulated numencally (solıd line) from the solution of (6.3) and from the asymptotic solution (dashed line) with $\arg (\kappa)=0, \arg (\phi)=-\pi / 2$ The labels (i) and (ii) on the graphs are for $\tilde{\sigma}=0$, and 5 respectively. (b) Imagınary part of $\tilde{A}^{\prime} / \tilde{A}$ against $Z$, other parameters are as in Figure 4(a). (c) Real part of $\tilde{A^{\prime}} / \bar{A}$ against $Z$, other parameters are as in Figure 4(a). 

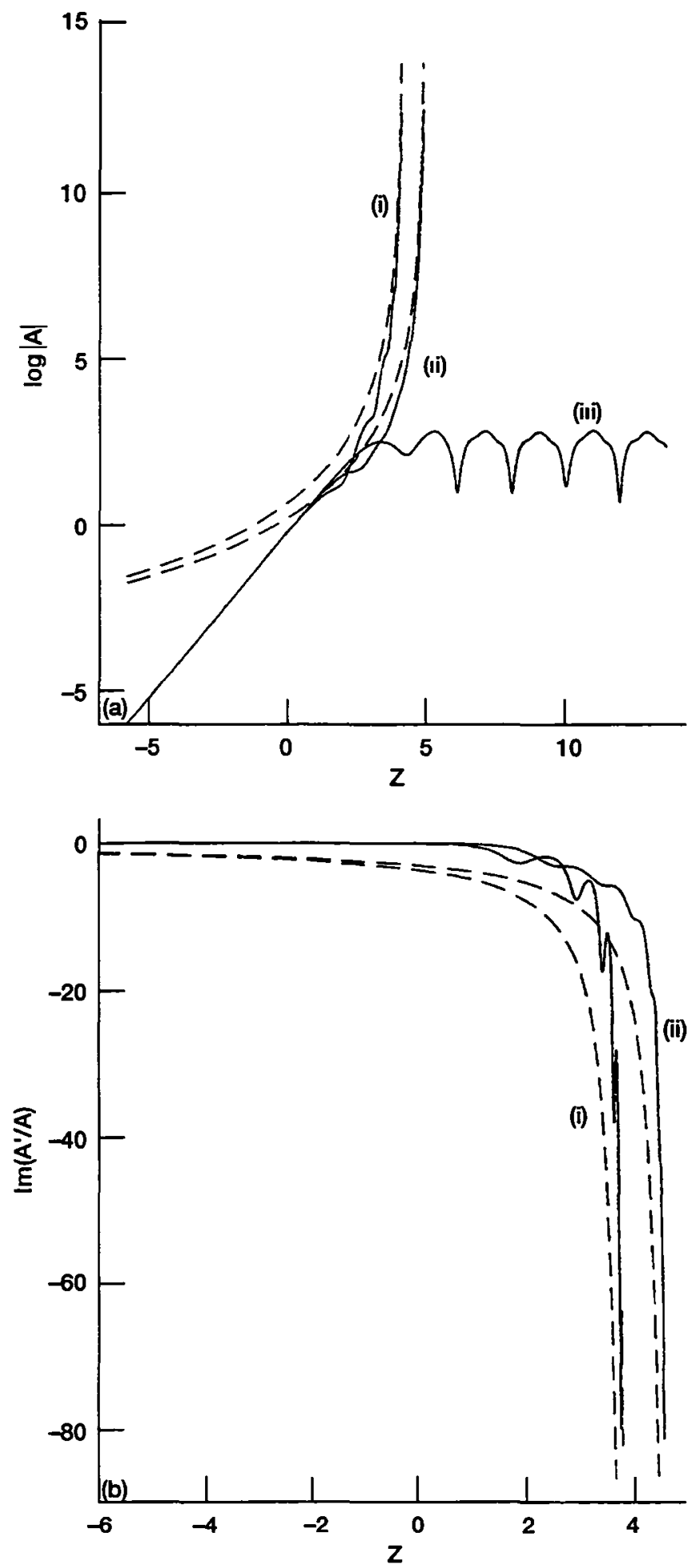

Figure 5.-(a) A plot of $\log |\tilde{A}|$ against $Z$ as calculated numencally (solid line) from the solution of (6.3) and from the asymptotic solution (dashed line) with $\arg (\kappa)=0 \arg (\phi)=-\pi / 4$. The labels (i), (ii) and (iii) on the graphs are for $\tilde{\sigma}=0,0.5$ and 5 respectively. (b) Imaginary part of $\tilde{A}^{\prime} / \bar{A}$ aganist $Z$, other parameters are as in Figures $5\left(\right.$ a). (c) Real part of $\tilde{A}^{\prime} / \tilde{A}$ against $Z$, other parameters are in Figure $5\left(\right.$ a). (d) Imaginary part of $\tilde{A}^{\prime} / \tilde{A}$ calculated numerically with $\tilde{\sigma}=5$, other parameters are as in Figure $5(\mathrm{a})$. (e) Real part of $\tilde{A}^{\prime} / \tilde{A}$ calculated numerically with $\tilde{\sigma}=5$, other parameters are as in Figure 5(a). (f) A plot of $|\tilde{A}|$ as calculated numencally with $\tilde{\sigma}=5$, other parameters are as in Figure 5(a). 

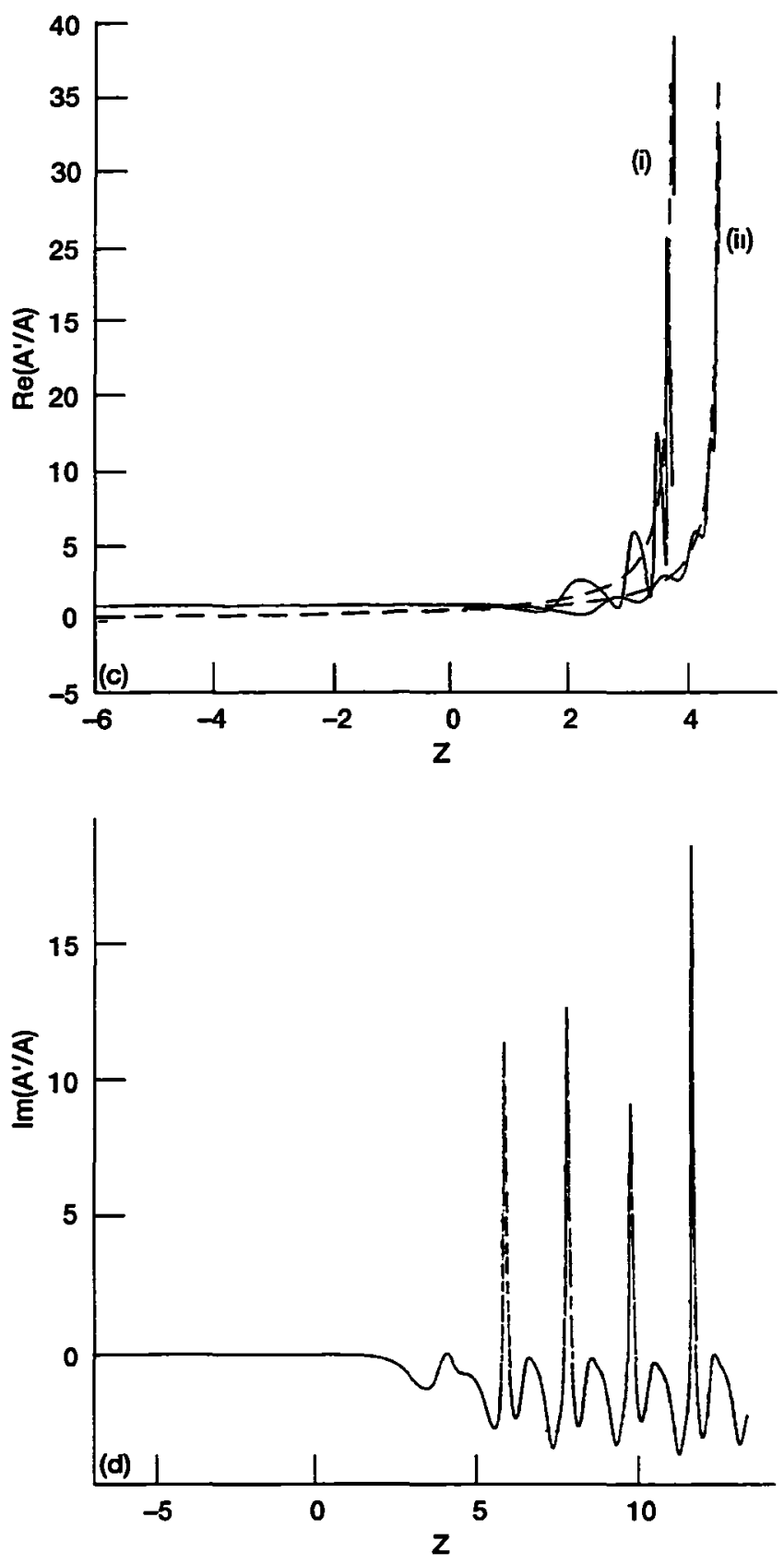

Figure 5.-Continued. (c) Real part of $\tilde{A}^{\prime} / \tilde{A}$ against $Z$, other parameters are as in Figure 5(a). (d) Imaginary part of $\bar{A}^{\prime} / \tilde{A}$ calulated numencally with $\tilde{\boldsymbol{\sigma}}=\mathbf{5}$, other parameters are as in Figure 5(a). 

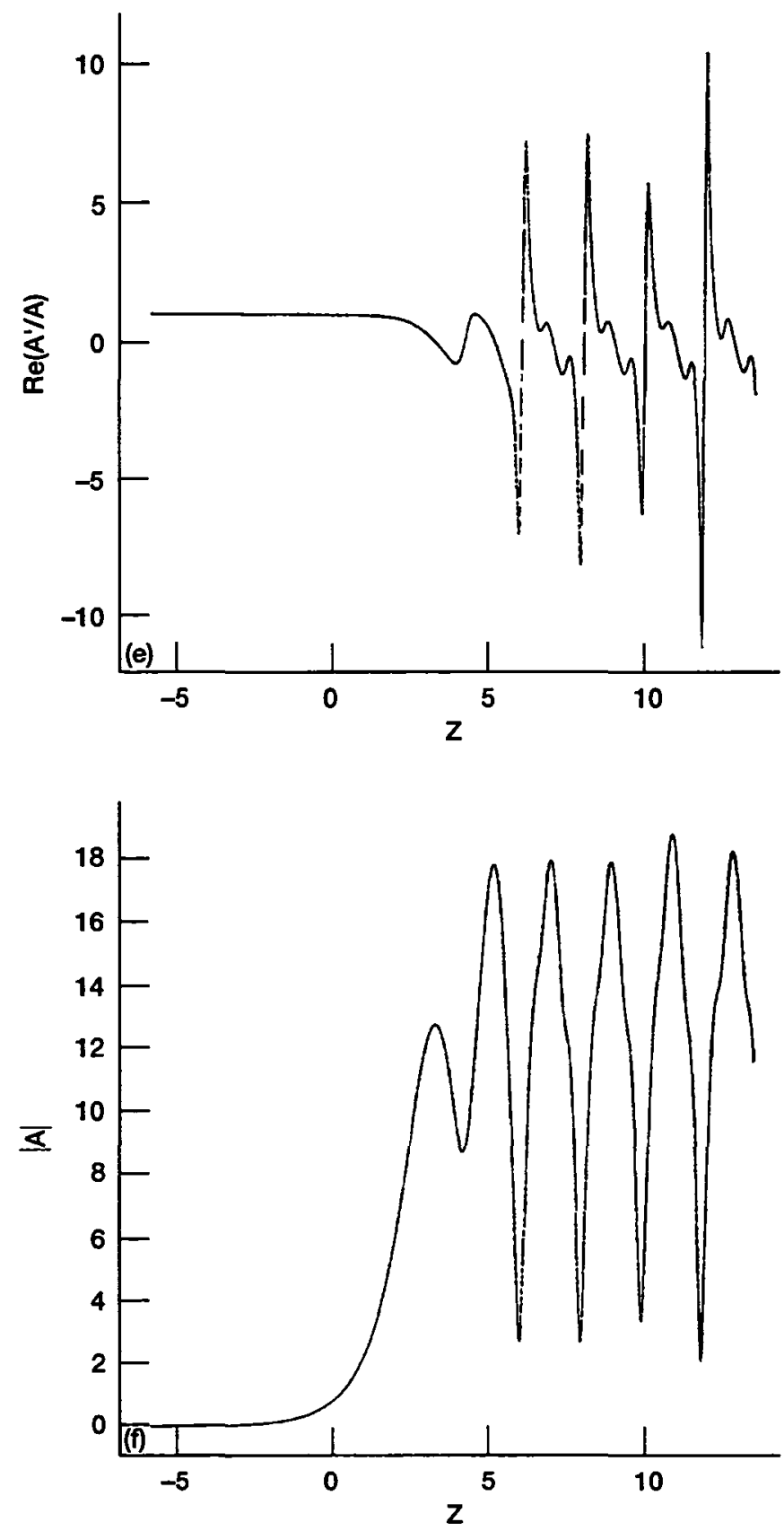

Figure 5.-Conciuded. (e) Real part of $\tilde{A}^{\prime} / \tilde{A}$ calculated numencally with $\tilde{\sigma}=5$, other parameters are as in Figure 5(a). (f) A plot of $|\tilde{A}|$ as calculated numencally with $\tilde{\sigma}=5$, other parameters are as in Figure 5(a) 

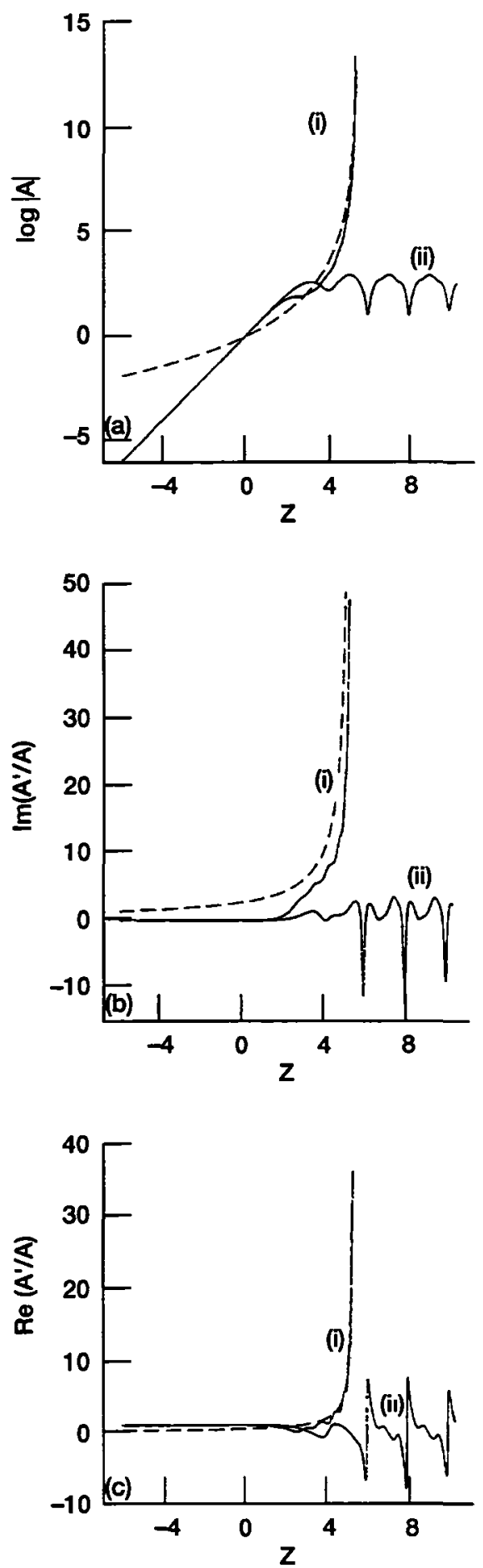

Figure 6.-(a) A plot of $\log |\tilde{\mathrm{A}}|$ against $Z$ as calculated numencally (solid line) from the solution of (6.3) and from the asymptotic solution (dashed line) with $\arg (\kappa)=0, \arg (\phi)=-\pi / 4$. The labels (i) and (ii) on the graphs are for $\tilde{\boldsymbol{\sigma}}=1$ and 5 respectively. (b) Imaginary part of $\tilde{A} / \tilde{A}$ against $Z$, other parameters are as in Figures 6(a). (c) Real part of $\tilde{A}^{\prime} / \tilde{A}$ against $Z$, other parameters are as in Figure 6(a). 


\begin{tabular}{|c|c|c|c|c|}
\hline \multicolumn{3}{|c|}{ REPORT DOCUMENTATION PAGE } & \multicolumn{2}{|r|}{$\begin{array}{l}\text { Form Approved } \\
\text { OMB No 0704-0188 }\end{array}$} \\
\hline \multicolumn{5}{|c|}{ 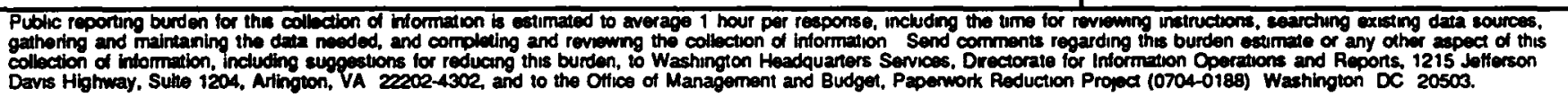 } \\
\hline 1. AGENCY USE ONLY (Leave blank) & \multicolumn{2}{|l|}{$\begin{array}{r}\text { 2. REPORTDATE } \\
\text { October } 1995\end{array}$} & \multicolumn{2}{|c|}{$\begin{array}{l}\text { D DATES COVERED } \\
\text { Contractor Report }\end{array}$} \\
\hline \multicolumn{3}{|c|}{$\begin{array}{l}\text { 4. TITLE AND SUBTILE } \\
\text { On the Nonlinear Evolution of a Stationary Cross-Flow Vortex in a Fully Three- } \\
\text { Dimensional Boundary Layer Flow }\end{array}$} & \multirow{2}{*}{\multicolumn{2}{|c|}{$\begin{array}{l}\text { 5. FUNDING NUMBERS } \\
\text { WU-505-90-5K } \\
\text { NCC3-370 }\end{array}$}} \\
\hline \multicolumn{3}{|l|}{$\begin{array}{l}\text { 6. AUTHOR(S) } \\
\text { J.S.B. Gajjar }\end{array}$} & & \\
\hline \multicolumn{3}{|c|}{$\begin{array}{l}\text { 7. PERFORMING OAGANIZATION NAME(S) AND ADDRESS(ES) } \\
\text { Instutute for Computatonal Mechanics in Propulsion } \\
22800 \text { Cedar Point Road } \\
\text { Cleveland, Ohio } 44142\end{array}$} & \multicolumn{2}{|c|}{$\begin{array}{l}\text { 8. PERFORMANG ORGANIZATION } \\
\text { REPORT NUMBER } \\
\text { E-9919 }\end{array}$} \\
\hline \multicolumn{3}{|c|}{$\begin{array}{l}\text { 9. SPONSORING/NONTORING AGENCY NAME(S) AND ADDRESS(ES) } \\
\text { National Aeronautics and Space Administration } \\
\text { Lewis Research Center } \\
\text { Cleveland, Ohio } 44135-3191\end{array}$} & \multicolumn{2}{|c|}{$\begin{array}{l}10 \text { SPONSORINGMONITORING } \\
\text { AGENCY REPORT NUMBER } \\
\\
\text { NASA CR-198405 } \\
\text { ICOMP-95-16 }\end{array}$} \\
\hline \multicolumn{5}{|c|}{$\begin{array}{l}\text { 11. SUPPLEMENTARY NOTES } \\
\text { J.S.B. Gayjar, Institute for Computational Mechanics in Propulsion and University of Manchester, Department of Math- } \\
\text { ematics, Manchester M13 9PL, United Kingdom. ICOMP Program Director, Louis A. Povinelli, organization code 2600, } \\
\text { (216) 433-5818. }\end{array}$} \\
\hline \multicolumn{3}{|c|}{$\begin{array}{l}122 \text { DISTRIBUTIONAVAILABILTYY STATEMENT } \\
\text { Unclassified - Unlimited } \\
\text { Subject Category } 34 \\
\text { Ths publication is avalable from the NASA Center for Aerospace Informaton, (301) 621-0390 }\end{array}$} & \multicolumn{2}{|c|}{ 12b. DISTRIBUTION CODE } \\
\hline \multicolumn{5}{|c|}{13 ABSTRACT (Maximum 200 words) } \\
\hline \multirow{2}{*}{\multicolumn{3}{|c|}{$\begin{array}{l}\text { 14. SUBJECT TERMS } \\
\text { Stability; Cross-flow vortex; Three-dımensional boundary layer }\end{array}$}} & & $\begin{array}{c}15 \text { NUMBER OF PAGES } \\
38\end{array}$ \\
\hline & & & & $\begin{array}{l}\text { 16. PRICE CODE } \\
\text { A03 }\end{array}$ \\
\hline $\begin{array}{l}\text { 17. SECURITY CLASSIFICATION } \\
\text { OF REPORT } \\
\text { Unclassified }\end{array}$ & $\begin{array}{l}\text { 18. SECURTY CLASSIFICATION } \\
\text { OF THIS PAGE } \\
\text { Unclassified }\end{array}$ & $\begin{array}{l}19 \text { SECURITY CLASSIFICA } \\
\text { OF ABSTRACT } \\
\text { Unclassified }\end{array}$ & & 20. LIMTTATKON OF ABSTRACT \\
\hline
\end{tabular}


End of Document 